\title{
Moving gap solitons in periodic potentials
}

\author{
Dmitry Pelinovsky*and Guido Schneider \\ Institut für Analysis, Dynamik und Modellierung Fakultät für Mathematik und Physik, \\ Universität Stuttgart, Pfaffenwaldring 57, D-70569 Stuttgart, Germany
}

November 11, 2018

\begin{abstract}
We address existence of moving gap solitons (traveling localized solutions) in the Gross-Pitaevskii equation with a small periodic potential. Moving gap solitons are approximated by the explicit localized solutions of the coupled-mode system. We show however that exponentially decaying traveling solutions of the Gross-Pitaevskii equation do not generally exist in the presence of a periodic potential due to bounded oscillatory tails ahead and behind the moving solitary waves. The oscillatory tails are not accounted in the coupled-mode formalism and are estimated by using techniques of spatial dynamics and local center-stable manifold reductions. Existence of bounded traveling solutions of the Gross-Pitaevskii equation with a single bump surrounded by oscillatory tails on a finite large interval of the spatial scale is proven by using these technique. We also show generality of oscillatory tails in other nonlinear equations with a periodic potential.
\end{abstract}

\section{Introduction}

Moving gap solitons are thought to be steadily traveling localized solutions of nonlinear partial differential equations with spatially periodic coefficients. The name of gap solitons comes from the fact that parameters of stationary localized solutions reside in the spectral gap of the associated Schrödinger operator. Existence of stationary solutions can be studied by separation of the time and space variables and reduction of the problem to an elliptic semi-linear equation. Since the variables are not separable for traveling solutions, a little is known about existence of moving gap solitons.

We address stationary and traveling localized solutions in the context of the Gross-Pitaevskii equation with an external periodic potential

$$
i E_{t}=-E_{x x}+V(x) E+\sigma|E|^{2} E,
$$

where $E(x, t): \mathbb{R} \times \mathbb{R}_{+} \mapsto \mathbb{C}, V(x): \mathbb{R} \mapsto \mathbb{R}$, and $\sigma= \pm 1$. The Gross-Pitaevskii equation (1.1) is derived for the mean-field amplitude of the Bose-Einstein condensate placed in the optical lattice trap $V(x)$, where $\sigma$ is the normalized scattering length [7]. Stationary solutions of the Gross-Pitaevskii equation are found from the solutions of the nonlinear differential equation

$$
\phi^{\prime \prime}(x)+\omega \phi(x)=V(x) \phi(x)+\sigma|\phi(x)|^{2} \phi(x),
$$

*On leave from Department of Mathematics, McMaster University, Hamilton, Ontario, Canada, L8S 4K1 
where $\phi(x): \mathbb{R} \mapsto \mathbb{C}, \omega \in \mathbb{R}$, and the exact reduction $E(x, t)=\phi(x) e^{-i \omega t}$ is used. Localized stationary solutions of the ODE problem (1.2) were proved to exist in [9].

Theorem 1 (Pankov, 05) Let $V(x)$ be a real-valued, bounded, and periodic potential. Let $\omega$ be in a finite gap of the purely continuous spectrum of $L=-\partial_{x}^{2}+V(x)$ in $L^{2}(\mathbb{R})$. There exists a nontrivial weak solution $\phi(x)$ in $H^{1}(\mathbb{R})$, which is (i) real-valued, (ii) continuous on $x \in \mathbb{R}$ and (iii) decays exponentially as $|x| \rightarrow \infty$.

In [10], we have obtained a more precise information on properties of the stationary solution $\phi(x)$ by working with a small potential $V(x)$, when the spectrum of $L$ exhibits a sequence of narrow gaps bifurcating near resonance points $\omega=\omega_{n}=\frac{n^{2}}{4}, n \in \mathbb{N}$. We have justified the use of the stationary coupled-mode equations which have been used in the physics literature [13] for explicit approximations of stationary gap solitons.

In this paper, we shall investigate whether the time-dependent coupled-mode equations can be used for approximation of moving gap solitons in the framework of the Gross-Pitaevskii equation (1.1). The coupled-mode equations are derived formally when the potential is represented by $V=\epsilon W(x)$, where $\epsilon$ is small parameter and $W(x)$ is a normalized $\epsilon$-independent function described as follows.

Assumption 1 Let $W(x)$ be a smooth $2 \pi$-periodic function with zero mean and symmetry $W(-x)=$ $W(x)$ on $x \in \mathbb{R}$. The Fourier series representation of $W(x)$ is

$$
W(x)=\sum_{m \in \mathbb{Z}} w_{2 m} e^{i m x}, \quad \text { such that } \sum_{m \in \mathbb{Z}}\left(1+m^{2}\right)^{s}\left|w_{2 m}\right|^{2}<\infty, \quad \forall s>\frac{1}{2},
$$

where $w_{0}=0$ and $w_{2 m}=w_{-2 m}=\bar{w}_{2 m}, \forall m \in \mathbb{N}$.

An asymptotic solution of the Gross-Pitaevskii equation (1.1) is represented in the form [4, 12]:

$$
E(x, t)=\epsilon^{1 / 2}\left[a(\epsilon x, \epsilon t) e^{\frac{i n x}{2}}+b(\epsilon x, \epsilon t) e^{-\frac{i n x}{2}}\right] e^{-\frac{i n^{2} t}{4}}+\mathrm{O}\left(\epsilon^{3 / 2}\right),
$$

where the vector function $(a, b): \mathbb{R} \times \mathbb{R}_{+} \mapsto \mathbb{C}^{2}$ satisfies the coupled-mode system

$$
\left\{\begin{aligned}
i\left(a_{T}+n a_{X}\right) & =w_{2 n} b+\sigma\left(|a|^{2}+2|b|^{2}\right) a \\
i\left(b_{T}-n b_{X}\right) & =w_{2 n} a+\sigma\left(2|a|^{2}+|b|^{2}\right) b
\end{aligned}\right.
$$

in slow variables $X=\epsilon x$ and $T=\epsilon t$. The system (1.5) admits a separation of variables [13]:

$$
a=\left(\frac{n+c}{n-c}\right)^{1 / 4} A(\xi) e^{-i \mu n \tau}, \quad b=\left(\frac{n-c}{n+c}\right)^{1 / 4} B(\xi) e^{-i \mu n \tau}
$$

where $|c|<n$, the new independent coordinates $(\xi, \tau)$ are given by the Lorentz transformation

$$
\xi=\frac{X-c T}{\sqrt{n^{2}-c^{2}}}, \quad \tau=\frac{T-c X}{\sqrt{n^{2}-c^{2}}},
$$

and the new functions $A(\xi)$ and $B(\xi)$ satisfy the coupled-mode system

$$
\left\{\begin{array}{c}
(n-c)\left(i A^{\prime}-w_{2 n} B\right)+\mu n(1-c n) A=\sigma\left[(n+c)|A|^{2}+2(n-c)|B|^{2}\right] A \\
-(n+c)\left(i B^{\prime}+w_{2 n} A\right)+\mu n(1+c n) B=\sigma\left[2(n+c)|A|^{2}+(n-c)|B|^{2}\right] B .
\end{array}\right.
$$


Since $|A|^{2}-|B|^{2}$ is constant in $\xi \in \mathbb{R}$ and the constant is zero for localized solutions at infinity $|\xi| \rightarrow \infty$, we can further represent the localized solution in the form

$$
A=\phi(\xi) e^{i \varphi(\xi)}, \quad B=\bar{\phi}(\xi) e^{i \varphi(\xi)}
$$

where the functions $\phi: \mathbb{R} \mapsto \mathbb{C}$ and $\varphi: \mathbb{R} \mapsto \mathbb{R}$ are solutions of the first-order equations

$$
\left\{\begin{aligned}
\varphi^{\prime} & =\frac{n c\left(\mu\left(1-n^{2}\right)-2 \sigma|\phi|^{2}\right)}{\left(n^{2}-c^{2}\right)} \\
i \phi^{\prime} & =w_{2 n} \bar{\phi}-\frac{\mu n^{2}\left(1-c^{2}\right)}{\left(n^{2}-c^{2}\right)} \phi+\sigma \frac{\left(3 n^{2}-c^{2}\right)}{\left(n^{2}-c^{2}\right)}|\phi|^{2} \phi .
\end{aligned}\right.
$$

The second equation of the system (1.10) is closed on $\phi(\xi)$ and the explicit localized solution for $c \neq 0$ can be found from the corresponding solution for $c=0$ [10]. For instance, if $\sigma=-1$ and $w_{2 n}>0$, the function $\phi(\xi)$ is found in the explicit form

$$
\phi=\sqrt{\frac{2\left(n^{2}-c^{2}\right)}{\left(3 n^{2}-c^{2}\right)}} \frac{\sqrt{w_{2 n}^{2}-\mu_{0}^{2}}}{\sqrt{w_{2 n}+\mu_{0}} \cosh \left(\sqrt{w_{2 n}^{2}-\mu_{0}^{2}} \xi\right)-i \sqrt{w_{2 n}-\mu_{0}} \sinh \left(\sqrt{w_{2 n}^{2}-\mu_{0}^{2}} \xi\right)},
$$

where $\mu_{0}=\mu \frac{n^{2}\left(1-c^{2}\right)}{\left(n^{2}-c^{2}\right)}$ and $\left|\mu_{0}\right|<w_{2 n}$. In the case $c=0$, we have $\mu_{0}=\mu$ and the condition $|\mu|<w_{2 n}$ indicates that the frequency parameter $\omega=\omega_{n}+\epsilon \mu$ of the stationary gap soliton with $c=0$ is chosen inside the newly formed gap of the continuous spectrum near the bifurcation point $\omega_{n}=\frac{n^{2}}{4}, n \in \mathbb{N}$ [10], such that

$$
\omega_{n}-\epsilon w_{2 n}<\omega<\omega_{n}+\epsilon w_{2 n} .
$$

The exact solution (1.11) can be extended easily to values $\sigma=-1$ and $w_{2 n}<0$. Given a localized solution for $\phi(\xi)$, we can integrate the first equation of the system (1.10) and obtain a linearly growing solution for $\varphi(\xi)$ :

$$
\varphi=\frac{n c}{\left(n^{2}-c^{2}\right)}\left(\mu\left(1-n^{2}\right) \xi-2 \sigma \int_{0}^{\xi}\left|\phi\left(\xi^{\prime}\right)\right|^{2} d \xi^{\prime}\right) .
$$

The trivial parameters of translations of solutions in $\xi$ and $\varphi$ are set to zero in the explicit solutions (1.11) and (1.13), such that the functions $A(\xi)$ and $B(\xi)$ given by the parametrization (1.9) satisfy the constraints $A(\xi)=\bar{A}(-\xi)$ and $B(\xi)=\bar{B}(-\xi)$.

Definition 1 The traveling solution of the coupled-mode system (1.5) is said to be a reversible homoclinic orbit if it decays to zero at infinity and satisfies the constraints $A(\xi)=\bar{A}(-\xi)$ and $B(\xi)=\bar{B}(-\xi)$ in parametrization (1.6)-(1.9).

We study persistence of the traveling solution (1.6) -(1.13) with $c \neq 0$ of the coupled-mode system (1.5) in the Gross-Pitaevskii equation (1.1). We show that the moving gap solitons have bounded oscillatory tails in the far-field profile of the scale $\epsilon^{N+1}$, which are small in amplitude of the order $\epsilon^{N+1 / 2}$ for any $N \geq 1$. These small oscillatory tails are not accounted in the coupled-mode system (1.5). The main result is formulated below.

Theorem 2 Let Assumption 1 be satisfied. Fix $n \in \mathbb{N}$, such that $w_{2 n} \neq 0$. Let $\omega=\frac{n^{2}}{4}+\epsilon \Omega$, such that $|\Omega|<\Omega_{0}=\left|w_{2 n}\right| \frac{\sqrt{n^{2}-c^{2}}}{n}$. Let $0<c<n$, such that $\frac{n^{2}+c^{2}}{2 c} \notin \mathbb{Z}^{\prime}$, where $\mathbb{Z}^{\prime}$ is a set of odd (even) numbers for odd (even) n. Fix $N \in \mathbb{N}$. For sufficiently small $\epsilon$, there are $\epsilon$-independent constants $L>0$ and 
$C>0$, such that there exists an infinite-dimensional, continuous family of traveling solutions of the Gross-Pitaevskii equation (1.1) in the form $E(x, t)=e^{-i \omega t} \psi(x, y)$, where $y=x-$ ct and the function $\psi(x, y)$ is periodic (anti-periodic) function of $x$ for even (odd) $n$, satisfying the reversibility constraint $\psi(x, y)=\bar{\psi}(x,-y)$, and the bound

$$
\left|\psi(x, y)-\epsilon^{1 / 2}\left[a_{\epsilon}(\epsilon y) e^{\frac{i n x}{2}}+b_{\epsilon}(\epsilon y) e^{-\frac{i n x}{2}}\right]\right| \leq C_{0} \epsilon^{N+1 / 2}, \quad \forall x \in \mathbb{R}, \forall y \in\left[-L / \epsilon^{N+1}, L / \epsilon^{N+1}\right],
$$

Here $a_{\epsilon}(Y)=a(Y)+\mathrm{O}(\epsilon)$ and $b_{\epsilon}(Y)=b(Y)+\mathrm{O}(\epsilon)$ on $Y=\epsilon y \in \mathbb{R}$ are exponentially decaying solutions as $|Y| \rightarrow \infty$, where $a(Y)$ and $b(Y)$ are solutions of the coupled-mode system (1.5) with $Y=X-c T$.

\section{Remark 1}

(a) The solution $\psi(x, y)$ is a bounded non-decaying function on a large finite interval

$$
y \in\left[-L / \epsilon^{N+1}, L / \epsilon^{N+1}\right] \subset \mathbb{R}
$$

but we do not claim that the solution $\psi(x, y)$ can be extended to a global bounded function on $y \in \mathbb{R}$.

(b) Since the homoclinic orbit $(a, b)$ of the coupled-mode system (1.5) is single-humped, the traveling solution $\psi(x, y)$ is represented by a single bump surrounded by bounded oscillatory tails.

(c) The solution $\left(a_{\epsilon}, b_{\epsilon}\right)$ is defined up to the terms of $\mathrm{O}\left(\epsilon^{N}\right)$ and it satisfies an extended coupled-mode system with a unique reversible single-humped homoclinic orbit.

Our work can be compared with three groups of papers. The first group covers rigorous justification of the validity of the coupled-mode system (1.5) for the system of cubic Maxwell equations [4] and for the Klein-Fock equation with quadratic nonlinearity [12. The bound on the error terms was proved for a finite time interval, which depends on $\epsilon$. By using this bound, one can see that the solution of the Gross-Pitaevskii equation (1.1) behaves as a moving gap soliton of the coupled-mode system (1.5) during the initial time evolution in $H^{1}(\mathbb{R})\left[4\right.$ or in $C_{b}^{0}(\mathbb{R})[12$. However, the error is not controlled on the entire time interval $t \in \mathbb{R}$ since other effects such as radiation due to interactions of the moving gap soliton with the stationary periodic potential can destroy steady propagation of gap solitons.

The second group of articles covers analysis of persistence of small-amplitude localized modulated pulses in nonlinear dispersive systems such as the Maxwell equations with periodic coefficients [1], the nonlinear wave equation [5], and the quasilinear wave equation [6]. Methods of spatial dynamics were applied in these works to show that a local center manifold spanned by oscillatory modes destroys exponential localization of the modulated pulses along the directions of the slow stable and unstable manifolds. As a result, the modulating pulse solutions decay in the spatial dynamics to small-amplitude oscillatory disturbances in the far-field regions.

The third group of papers addresses propagation of a moving solitary wave in a periodic potential $V(x)$ of a large period (see review in [11]). An effective particle equation is derived from the focusing Gross-Pitaevskii equation (1.1) with $\sigma=-1$ by an heuristic asymptotic expansion. The particle equation describes a steady propagation of the moving solitary wave with $\omega<0$, which corresponds to the semi-infinite gap of the periodic potential. Radiation effects appear beyond all orders of the asymptotic expansion. They have been incorporated in the asymptotic formalism by using perturbation theory based on the inverse scattering transform [8]. The same methods were applied to the finiteperiod and small-period potentials [11]. Unfortunately, this group of article does not connect individual 
results in a complete rigorous theory of the time evolution of a solitary wave in a periodic potential, although it does gives a good intuition on what to expect from the time evolution.

Our article is structured as follows. Section 2 reformulates the existence problem for moving gap solitons as the spatial dynamical system. Section 3 presents the Hamiltonian structure for the spatial dynamical system and normal coordinates of the Hamiltonian system. Section 4 describes a transformation of the Hamiltonian system to the normal form and gives a proof of persistence of a reversible homoclinic orbit in the extended coupled-mode system. Section 5 presents a construction of a local center-saddle manifold which concludes the proof of Theorem 2. Section 6 discusses other models for moving gap solitons with oscillatory tails.

\section{Spatial dynamics formulation}

We look for traveling solutions of the Gross-Pitaevskii equation (1.1) in the form

$$
E(x, t)=e^{-i \omega t} \psi(x, y), \quad y=x-c t,
$$

where $\omega$ is a parameter of gap solitons and the coordinates $(x, y)$ are linearly independent if $c \neq 0$. For simplicity, we only consider the case $c>0$. The envelope function $\psi(x, y)$ satisfies the partial differential equation

$$
\left(\omega-i c \partial_{y}+\partial_{x}^{2}+2 \partial_{x} \partial_{y}+\partial_{y}^{2}\right) \psi(x, y)=\epsilon W(x) \psi(x, y)+\sigma|\psi(x, y)|^{2} \psi(x, y) .
$$

At this stage, the equation (2.2) is equivalent to the original equation (1.1) if $c \neq 0$. We shall however specify the class of functions $\psi(x, y)$ to accommodate the moving gap solitons according to their leadingorder representation given by (1.4), (1.6), and (1.7). In particular, we consider either periodic (for even $n$ ) or anti-periodic (for odd $n$ ) functions $\psi(x, y)$ in variable $x \in[0,2 \pi]$ and look for decaying or bounded solutions $\psi(x, y)$ in variable $y \in \mathbb{R}$. Such solutions can be described by using the formalism of spatial dynamical systems [5, 6]. We make use of the periodic or anti-periodic conditions in variable $x$ and represent the solution $\psi(x, y)$ in the form

$$
\psi(x, y)=\sqrt{\epsilon} \sum_{m \in \mathbb{Z}^{\prime}} \psi_{m}(y) e^{\frac{i}{2} m x}, \quad \psi_{m}(y)=\frac{1}{2 \pi \sqrt{\epsilon}} \int_{0}^{2 \pi} \psi(x, y) e^{-\frac{i}{2} m x} d x, \quad m \in \mathbb{Z}^{\prime},
$$

where the factor $\sqrt{\epsilon}$ is used for the convenience and the set $\mathbb{Z}^{\prime}$ contains even numbers if $\psi(x, y)$ is periodic in $x$ and odd numbers if $\psi(x, y)$ is anti-periodic in $x$. The series representation (2.3) transforms the PDE system (2.2) to the nonlinear system of coupled ODEs

$$
\begin{array}{r}
\psi_{m}^{\prime \prime}(y)+i(m-c) \psi_{m}^{\prime}(y)+\left(\omega-\frac{m^{2}}{4}\right) \psi_{m}(y)=\epsilon \sum_{m_{1} \in \mathbb{Z}^{\prime}} w_{m-m_{1}} \psi_{m_{1}}(y) \\
+\epsilon \sigma \sum_{m_{1} \in \mathbb{Z}^{\prime}} \sum_{m_{2} \in \mathbb{Z}^{\prime}} \psi_{m_{1}}(y) \bar{\psi}_{-m_{2}}(y) \psi_{m-m_{1}-m_{2}}(y), \quad m \in \mathbb{Z}^{\prime}
\end{array}
$$

The left-hand-side of the system (2.4) represents a linearized system at the zero solution for $\epsilon=0$. Since the linearized system at $\epsilon=0$ has a diagonal structure on $m \in \mathbb{Z}^{\prime}$, its solutions are given by the 
eigenmodes $\psi_{m^{\prime}}(y)=e^{\kappa_{m} y} \delta_{m, m^{\prime}}$ with $m, m^{\prime} \in \mathbb{Z}^{\prime}$, where the values of $\kappa_{m}$ are determined by the roots of quadratic equations

$$
\kappa=\kappa_{m}: \quad \kappa^{2}+i(m-c) \kappa+\omega-\frac{m^{2}}{4}=0, \quad m \in \mathbb{Z}^{\prime} .
$$

The zero root $\kappa=0$ exists if and only if $\omega=\omega_{n}=\frac{n^{2}}{4}$ for any fixed $n \in \mathbb{Z}$. The zero root has multiplicity two for $m= \pm n$ if $n \in \mathbb{N}$ and $c \neq \pm n$. The special value $\omega=\omega_{n}$ corresponds to the bifurcation of periodic or anti-periodic solutions as well as of the stationary gap solitons with $c=0$ [10]. We note that the values of $n$ determine the choice for the set $\mathbb{Z}^{\prime}$ : it includes even (odd) numbers if $n$ is even (odd). We shall hence focus on the bifurcation case $\omega=\omega_{n}$, when the two roots of the quadratic equations (2.5) are represented explicitly as follows

$$
\omega=\omega_{n}: \quad \kappa=\kappa_{m}^{ \pm}=\frac{i(c-m) \pm \sqrt{2 c m-n^{2}-c^{2}}}{2}, \quad m \in \mathbb{Z}^{\prime} .
$$

When $m>m_{0}=\left[\frac{n^{2}+c^{2}}{2 c}\right]$, where the notation $[a]^{\prime}$ denotes the integer part of the number $a$ in the set $\mathbb{Z}^{\prime}$, all roots are complex-valued with $\operatorname{Re}\left(\kappa_{m}^{ \pm}\right)= \pm \frac{1}{2} \sqrt{2 c m-n^{2}-c^{2}} \neq 0$ and $\operatorname{Im}\left(\kappa_{m}^{ \pm}\right)=\frac{c-m}{2}$. When $m \leq m_{0}$, al roots $\kappa$ are purely imaginary with $\kappa_{m}^{ \pm}=i k_{m}^{ \pm}$and $k_{m}^{ \pm}=\frac{(c-m) \pm \sqrt{n^{2}+c^{2}-2 c m}}{2}$.

Lemma 1 Let $\omega=\omega_{n}, n \in \mathbb{N}$ and $c>0$, such that $\frac{n^{2}+c^{2}}{2 c} \notin \mathbb{Z}^{\prime}$. Then,

(i) The phase space of the linearized system 2.4 at the zero solution for $\epsilon=0$ decomposes into a direct sum of subspaces $E^{s} \oplus E^{u} \oplus E^{c^{+}} \oplus E^{c^{-}}$, where

$$
E^{s}=\oplus_{m>m_{0}} E_{m}^{+}, \quad E^{u}=\oplus_{m>m_{0}} E_{m}^{-}, \quad E^{c^{+}}=\oplus_{m \leq m_{0}} E_{m}^{+}, \quad E^{c^{-}}=\oplus_{m \leq m_{0}} E_{m}^{-} .
$$

The subspace $E_{m}^{ \pm}$consists of the eigenspace associated with the $m$-th Fourier component of the solution (2.3) corresponding to the root $\kappa=\kappa_{m}^{ \pm}$in (2.6).

(ii) The zero root $\kappa=0$ is semi-simple of multiplicity two. The purely imaginary roots $\kappa \in i \mathbb{R}$ are semi-simple of the maximal multiplicity three. All other roots $\kappa \in \mathbb{C}$ are simple.

Proof. It follows from the quadratic equation (2.5) that a root $\kappa=\kappa_{0}$ is double if $\kappa_{0}=\frac{i(c-m)}{2}$, which implies that $m=\frac{n^{2}+c^{2}}{2 c}$. Under the non-degeneracy constraint $\frac{n^{2}+c^{2}}{2 c} \notin \mathbb{Z}^{\prime}$, all roots $\kappa$ are semi-simple. When $m>m_{0}=\left[\frac{n^{2}+c^{2}}{2 c}\right]^{\prime}$, all roots are complex-valued and simple, such that $E^{s}$ and $E^{u}$ are stable and unstable manifolds of the linearized system at the zero solution for $\epsilon=0$.

When $m \leq m_{0}$, all roots $\kappa$ are purely imaginary, such that $E^{c^{+}} \oplus E^{c^{-}}$is a center manifold of the linearized system for $\epsilon=0$. It is obvious that $\kappa_{m}^{+}$increases as $m$ decreases, while $\kappa_{m}^{-}$decreases for $m_{1}<m \leq m_{0}$ and increases for $m \leq m_{1}$ as $m$ decreases, where $m_{1}=\left[\frac{n^{2}}{2 c}\right]^{\prime}$. Therefore, the purely imaginary roots may have the maximal multiplicity three. The zero eigenvalue has however multiplicity two since the two modes $m=n$ and $m=-n$ have simple zero eigenvalues and other modes have no zero eigenvalues.

Lemma 2 Let $\omega=\omega_{n}, n \in \mathbb{N}$ and $0<c<n$. If $c$ is irrational, all non-zero roots $\kappa$ of the quadratic equations (2.5) are simple. 
Proof. By Lemma 1, only imaginary roots $\kappa$ can be semi-simple. Let two roots $\kappa$ coincide for $m \leq m_{0}$ and $l \leq m_{0}$. Then, $\kappa=-i \frac{m+l}{4}$ and $(m, l)$ satisfies the equation

$$
(m-l)^{2}+4 c(m+l)-4 n^{2}=0, \quad m \leq m_{0}, l \leq m_{0} .
$$

If $c$ is irrational, equation (2.8) has no solutions for integers $m$ and $l$. Therefore, all non-zero roots $\kappa$ are simple.

Example 1 Figure 1(a) illustrates the distribution of imaginary roots $\kappa_{m}^{ \pm}=i k_{m}^{ \pm}, m \leq m_{0}$ for $c=\frac{1}{\sqrt{2}}$ and $n=1$. Although all imaginary roots are simple for this (irrational) value of $c$, the purely imaginary roots $\kappa$ can approach to each other arbitrarily close. Figure 1(b) shows a similar distribution for $c=\frac{1}{2}$ and $n=1$. It follows from equation (2.8) that an infinite sequence of semi-simple roots exists for this (rational) value of $c$.
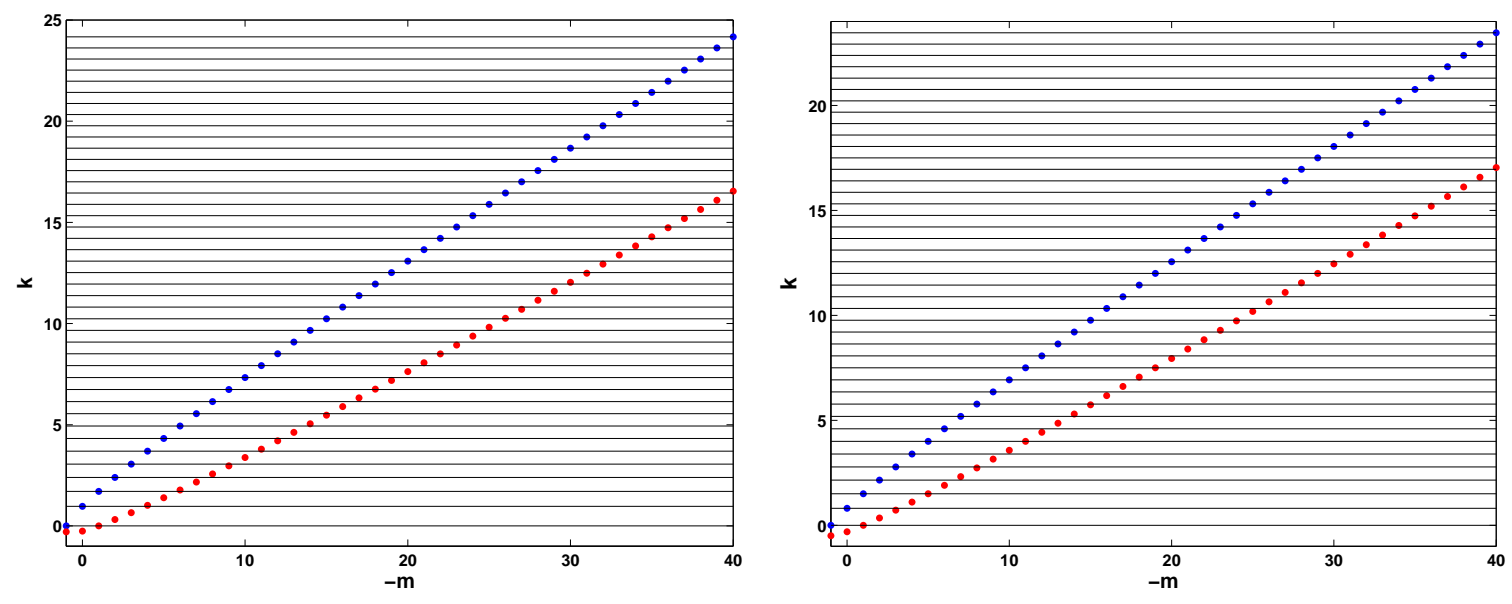

Figure 1: Purely imaginary roots $\kappa_{m}^{ \pm}=i k_{m}^{ \pm}$versus $m \leq m_{0}$ for $n=1, c=\frac{1}{\sqrt{2}}$ (left) and for $n=1$, $c=\frac{1}{2}$ (right). Blue dots correspond to $k_{m}^{+}$and red dots correspond to $k_{m}^{-}$.

Remark 2 When $c=0$, the formalism of spatial dynamical systems fails. Indeed, the linearized system (2.4) for $\epsilon=0$ and $c=0$ has a set of semi-simple eigenvalues of multiplicity two at $\kappa= \pm i k$, $k \in \mathbb{Z}$. However, all non-zero eigenvalues are equivalent to the zero eigenvalue due to existence of infinitely many symmetries for the system (2.4): $\psi_{m}(y) \mapsto \psi_{m+m^{\prime}}(y) e^{\frac{i m^{\prime} y}{2}}, \forall m^{\prime} \in \mathbb{Z}^{\prime}$. Therefore, the behavior of all modes with $m \in \mathbb{Z}^{\prime} \backslash\{n,-n\}$ repeat the behavior of the two resonant modes with $m= \pm n$ and only these two modes are relevant for existence of localized solutions for $\epsilon \neq 0$ and $c=0$. The failure of the spatial dynamics formulation is related to the fact that $y=x$ for $c=0$, such that the second-order ODE (1.2) can not be replaced by the PDE (2.2) and hence it can not be written as a system of infinitely many second-order ODEs (2.4).

Lemma 3 Consider a linear inhomogeneous equation

$$
\left(\partial_{y}^{2}+i(m-c) \partial_{y}+\frac{n^{2}-m^{2}}{4}\right) \psi_{m}(y)=-F_{m}(y), \quad \forall m>m_{0},
$$


where $F_{m} \in C_{b}^{0}(\mathbb{R})$. There exists a unique solution $\psi_{m} \in C_{b}^{0}(\mathbb{R})$, such that $\left\|\psi_{m}\right\|_{C_{b}^{0}(\mathbb{R})} \leq C\left\|F_{m}\right\|_{C_{b}^{0}(\mathbb{R})}$ for some $C>0$.

Proof. Let $\psi_{m}=e^{-i \alpha y} \varphi_{m}(y)$ with $\alpha=\frac{m-c}{2}$ and rewrite the linear equation (2.9) in the equivalent form

$$
\left(\beta^{2}-\partial_{y}^{2}\right) \varphi_{m}(y)=F_{m}(y) e^{i \alpha y}, \quad \beta^{2}=\frac{2 c m-n^{2}-c^{2}}{4}>0 .
$$

Since solutions of the homogeneous equation are exponentially decaying and growing as $\varphi_{m} \sim e^{ \pm \beta y}$, there exists a unique bounded solution of the inhomogeneous equation in the integral form

$$
\varphi_{m}(y)=\frac{1}{2 \beta} \int_{-\infty}^{\infty} e^{-\beta\left|y-y^{\prime}\right|} F_{m}\left(y^{\prime}\right) e^{i \alpha y^{\prime}} d y^{\prime}
$$

such that $\left\|\varphi_{m}\right\|_{C_{b}^{0}(\mathbb{R})} \leq \frac{1}{\beta^{2}}\left\|F_{m}\right\|_{C_{b}^{0}(\mathbb{R})}$.

Remark 3 By using Lemma 3 and the Implicit Function Theorem in suitable vector spaces, one can solve equations of the system (2.4) for $m>m_{0}$ and parameterize all components $\psi_{m}(y)$ with $m>m_{0}$ by bounded components $\psi_{m}(y)$ with $m \leq m_{0}$ for sufficiently small $\epsilon$. However, we do not perform this elimination at this stage, since we are going to rewrite the system (2.4) as a Hamiltonian dynamical system and use a formalism of near-identity transformations and normal forms, which works easier if the symplectic structure of the Hamiltonian system is local.

\section{Hamiltonian formalism and normal coordinates}

We rewrite the system of second-order equations (2.4) as the system of first-order equations which admits a symplectic Hamiltonian structure. Let $\omega=\frac{n^{2}}{4}+\epsilon \Omega$, where $n \in \mathbb{N}$ and $\Omega$ is a free parameter. Let $\phi_{m}(y)=\psi_{m}^{\prime}(y)-\frac{i}{2}(c-m) \psi_{m}(y)$ for all $m \in \mathbb{Z}^{\prime}$. The system (2.4) is equivalent to the first-order system

$$
\left\{\begin{aligned}
\frac{d \psi_{m}}{d y}= & \phi_{m}+\frac{i}{2}(c-m) \psi_{m} \\
\frac{d \phi_{m}}{d y}= & -\frac{1}{4}\left(n^{2}+c^{2}-2 c m\right) \psi_{m}+\frac{i}{2}(c-m) \phi_{m}-\epsilon \Omega \psi_{m}+\epsilon \sum_{m_{1} \in \mathbb{Z}^{\prime}} w_{m-m_{1}} \psi_{m_{1}} \\
& \quad+\epsilon \sigma \sum_{m_{1} \in \mathbb{Z}^{\prime}} \sum_{m_{2} \in \mathbb{Z}^{\prime}} \psi_{m_{1}} \bar{\psi}_{-m_{2}} \psi_{m-m_{1}-m_{2}} .
\end{aligned}\right.
$$

Let bolded symbol $\boldsymbol{\psi}$ denote a vector consisting of elements of the set $\left\{\psi_{m}\right\}_{m \in \mathbb{Z}^{\prime}}$. The variables $\{\boldsymbol{\psi}, \boldsymbol{\phi}, \overline{\boldsymbol{\psi}}, \overline{\boldsymbol{\phi}}\}$ are canonical and the system (3.1) is equivalent to the Hamilton's equations of motion

$$
\frac{d \psi_{m}}{d y}=\frac{\partial H}{\partial \bar{\phi}_{m}}, \quad \frac{d \phi_{m}}{d y}=-\frac{\partial H}{\partial \bar{\psi}_{m}}, \quad m \in \mathbb{Z}^{\prime},
$$

where $H=H(\boldsymbol{\psi}, \boldsymbol{\phi}, \overline{\boldsymbol{\psi}}, \overline{\boldsymbol{\phi}})$ is the Hamiltonian function given by

$$
\begin{aligned}
H= & \sum_{m \in \mathbb{Z}^{\prime}}\left[\left|\phi_{m}\right|^{2}+\frac{1}{4}\left(n^{2}+c^{2}-2 c m\right)\left|\psi_{m}\right|^{2}+\frac{i}{2}(c-m)\left(\psi_{m} \bar{\phi}_{m}-\bar{\psi}_{m} \phi_{m}\right)+\epsilon \Omega\left|\psi_{m}\right|^{2}\right] \\
& -\epsilon \sum_{m \in \mathbb{Z}^{\prime}} \sum_{m_{1} \in \mathbb{Z}^{\prime}} w_{m-m_{1}} \psi_{m_{1}} \bar{\psi}_{m}-\frac{\epsilon \sigma}{2} \sum_{m \in \mathbb{Z}^{\prime}} \sum_{m_{1} \in \mathbb{Z}^{\prime}} \sum_{m_{2} \in \mathbb{Z}} \psi_{m_{1}} \bar{\psi}_{-m_{2}} \psi_{m-m_{1}-m_{2}} \bar{\psi}_{m} .
\end{aligned}
$$


Let us define the discrete weighted $l^{2}$-space by its norm

$$
\forall \mathbf{u} \in l_{s}^{2}\left(\mathbb{Z}^{\prime}\right): \quad\|\mathbf{u}\|_{l_{s}^{2}}^{2}=\sum_{m \in \mathbb{Z}^{\prime}}\left(1+m^{2}\right)^{s}\left|u_{m}\right|^{2}<\infty
$$

Since $l_{s}^{2}\left(\mathbb{Z}^{\prime}\right)$ is Banach algebra for $s>\frac{1}{2}$, the convolution sums in the nonlinear system (3.1) are closed if $\mathbf{W} \in l_{s}^{2}(\mathbb{Z})$ (Assumption 1) and $\boldsymbol{\psi} \in l_{s}^{2}\left(\mathbb{Z}^{\prime}\right)$ for $s>\frac{1}{2}$. Due to the unbounded linear part, the vector field of the system (3.1) map a domain in $D$ and to a range in $X$, where $D$ and $X$ are given by

$$
D=\left\{(\boldsymbol{\psi}, \boldsymbol{\phi}, \overline{\boldsymbol{\psi}}, \overline{\boldsymbol{\phi}}) \in l_{s+1}^{2}\left(\mathbb{Z}^{\prime}, \mathbb{C}^{4}\right)\right\}, \quad X=\left\{(\boldsymbol{\psi}, \boldsymbol{\phi}, \overline{\boldsymbol{\psi}}, \overline{\boldsymbol{\phi}}) \in l_{s}^{2}\left(\mathbb{Z}^{\prime}, \mathbb{C}^{4}\right)\right\},
$$

for any $s>\frac{1}{2}$. We note that $D \subset X$ and that $X$ can be chosen as the phase space of the Hamiltonian system (3.2).

If $W(-x)=W(x), \forall x \in \mathbb{R}$ (Assumption 1), then $w_{2 m}=w_{-2 m}, \forall m \in \mathbb{Z}$. In this case, the Hamiltonian system (3.1) is reversible and its solutions are invariant under the transformation

$$
\psi(y) \mapsto \bar{\psi}(-y), \quad \phi(y) \mapsto-\bar{\phi}(-y) .
$$

In addition, the Hamiltonian function (3.3) is invariant with respect to the gauge transformation

$$
\boldsymbol{\psi}(y) \mapsto e^{i \alpha} \boldsymbol{\psi}(y), \quad \boldsymbol{\phi}(y) \mapsto e^{i \alpha} \boldsymbol{\phi}(y), \quad \forall \alpha \in \mathbb{R} .
$$

Let us define the domain for reversible solutions by

$$
D_{r}=\{(\boldsymbol{\psi}, \boldsymbol{\phi}, \overline{\boldsymbol{\psi}}, \overline{\boldsymbol{\phi}}) \in D: \quad \boldsymbol{\psi}(-y)=\overline{\boldsymbol{\psi}}(y), \quad \phi(-y)=-\overline{\boldsymbol{\phi}}(y)\} .
$$

If a local solution $(\boldsymbol{\psi}(y), \phi(y))$ of the system (3.1) is constructed on $y \in \mathbb{R}_{+}$and it intersects at $y=0$ with the reversibility constraint

$$
\Sigma_{r}=\{(\boldsymbol{\psi}, \boldsymbol{\phi}, \overline{\boldsymbol{\psi}}, \overline{\boldsymbol{\phi}}) \in D: \quad \operatorname{Im} \boldsymbol{\psi}=0, \quad \operatorname{Re} \phi=0\},
$$

then the solution $(\boldsymbol{\psi}(y), \boldsymbol{\phi}(y))$ is extended to a global reversible solution in $D_{r}$ on $y \in \mathbb{R}$ by using the reversibility transformation (3.6). The global reversible solution does not have an arbitrary parameter $\alpha$ induced by the gauge transformation (3.7).

In order to construct non-trivial bounded solutions of the Hamiltonian system (3.1), we shall introduce normal coordinates for the infinite-dimensional stable, unstable and center manifolds of the linearized Hamiltonian system (3.1) at the zero solution for $\epsilon=0$ (Lemma 1). Let $\mathbb{Z}_{-}^{\prime}=\left\{m \in \mathbb{Z}^{\prime}\right.$ : $\left.m \leq m_{0}\right\}$ and $\mathbb{Z}_{+}^{\prime}=\left\{m \in \mathbb{Z}^{\prime}: m>m_{0}\right\}$. For the center manifold of the linearized Hamiltonian system, we set

$$
m \in \mathbb{Z}_{-}^{\prime}: \quad \psi_{m}=\frac{c_{m}^{+}(y)+c_{m}^{-}(y)}{\sqrt[4]{n^{2}+c^{2}-2 c m}}, \quad \phi_{m}=\frac{i}{2} \sqrt[4]{n^{2}+c^{2}-2 c m}\left[c_{m}^{+}(y)-c_{m}^{-}(y)\right]
$$

For the stable and unstable manifolds of the linearized Hamiltonian system, we set

$$
m \in \mathbb{Z}_{+}^{\prime}: \quad \psi_{m}=\frac{c_{m}^{+}(y)+c_{m}^{-}(y)}{\sqrt[4]{2 c m-n^{2}-c^{2}}}, \quad \phi_{m}=\frac{1}{2} \sqrt[4]{2 c m-n^{2}-c^{2}}\left[c_{m}^{+}(y)-c_{m}^{-}(y)\right]
$$


By using the normal coordinates (3.10) -(3.11), the Hamiltonian function (3.3) transforms to the new form

$$
\begin{aligned}
H= & \sum_{m \in \mathbb{Z}_{-}^{\prime}}\left(k_{m}^{+}\left|c_{m}^{+}\right|^{2}-k_{m}^{-}\left|c_{m}^{-}\right|^{2}\right)+\sum_{m \in \mathbb{Z}_{+}^{\prime}}\left(\kappa_{m}^{-} c_{m}^{-} \bar{c}_{m}^{+}-\kappa_{m}^{+} c_{m}^{+} \bar{c}_{m}^{-}\right) \\
& +\epsilon \Omega \sum_{m \in \mathbb{Z}^{\prime}} \frac{\left|c_{m}^{+}+c_{m}^{-}\right|^{2}}{\sqrt{\left|n^{2}+c^{2}-2 c m\right|}}-\epsilon \sum_{m \in \mathbb{Z}^{\prime}} \sum_{m_{1} \in \mathbb{Z}^{\prime}} w_{m, m_{1}}\left(c_{m_{1}}^{+}+c_{m_{1}}^{-}\right)\left(\bar{c}_{m}^{+}+\bar{c}_{m}^{-}\right) \\
& -\frac{\epsilon \sigma}{2} \sum_{m \in \mathbb{Z}^{\prime}} \sum_{m_{1} \in \mathbb{Z}^{\prime}} \sum_{m_{2} \in \mathbb{Z}} g_{m, m_{1}, m_{2}}\left(c_{m_{1}}^{+}+c_{m_{1}}^{-}\right)\left(\bar{c}_{-m_{2}}^{+}+\bar{c}_{-m_{2}}^{-}\right)\left(c_{m-m_{1}-m_{2}}^{+}+c_{m-m_{1}-m_{2}}^{-}\right)\left(\bar{c}_{m}^{+}+\bar{c}_{m}^{-}\right),
\end{aligned}
$$

where

$$
\begin{aligned}
& k_{m}^{ \pm}=\frac{c-m \pm \sqrt{n^{2}+c^{2}-2 c m}}{2}, \quad m \in \mathbb{Z}_{-}^{\prime}, \\
& \kappa_{m}^{ \pm}=\frac{i(c-m) \pm \sqrt{2 c m-n^{2}-c^{2}}}{2}, \quad m \in \mathbb{Z}_{+}^{\prime}, \\
& w_{m, m_{1}}=\frac{w_{m-m_{1}}}{\sqrt[4]{\left|n^{2}+c^{2}-2 c m\right|\left|n^{2}+c^{2}-2 c m_{1}\right|}}, \quad m, m_{1} \in \mathbb{Z}^{\prime}
\end{aligned}
$$

and

$$
g_{m, m_{1}, m_{2}}=\frac{1}{\sqrt[4]{\left|n^{2}+c^{2}-2 c m_{1}\right|\left|n^{2}+c^{2}+2 c m_{2}\right|\left|n^{2}+c^{2}-2 c\left(m-m_{1}-m_{2}\right)\right|\left|n^{2}+c^{2}-2 c m\right|}},
$$

for all $m, m_{1}, m_{2} \in \mathbb{Z}^{\prime}$. The quadratic part of the Hamiltonian function in (3.12) for $\epsilon=0$ is diagonal in normal coordinates for $m \in \mathbb{Z}_{-}^{\prime}$ and it is block-diagonal for $m \in \mathbb{Z}_{+}^{\prime}$. The Hamiltonian equations of motions (3.2) transform in new canonical variables to the form

$$
\frac{d c_{m}^{+}}{d y}=i \frac{\partial H}{\partial \bar{c}_{m}^{+}}, \quad \frac{d c_{m}^{-}}{d y}=-i \frac{\partial H}{\partial \bar{c}_{m}^{-}}, \quad m \in \mathbb{Z}_{-}^{\prime}
$$

and

$$
\frac{d c_{m}^{+}}{d y}=-\frac{\partial H}{\partial \bar{c}_{m}^{-}}, \quad \frac{d c_{m}^{-}}{d y}=\frac{\partial H}{\partial \bar{c}_{m}^{+}}, \quad m \in \mathbb{Z}_{+}^{\prime} .
$$

Since the convolution sums on $\left\{c_{m}^{ \pm}\right\}_{m \in \mathbb{Z}^{\prime}}$ involve decaying weights as $|m| \rightarrow \infty$ and the linear unbounded part on $\left\{c_{m}\right\}_{m \in \mathbb{Z}^{\prime}}$ is linear in $|m|\left(k_{m}^{ \pm}\right.$and $\kappa_{m}^{ \pm}$grow linearly as $\left.|m| \rightarrow \infty\right)$, the vector field of the system (3.13) -(3.14) has a modified domain $D^{\prime}$ and range $X^{\prime}$, which are given by

$$
D^{\prime}=\left\{\left(\mathbf{c}^{+}, \mathbf{c}^{-}, \overline{\mathbf{c}}^{+}, \overline{\mathbf{c}}^{-}\right) \in l_{s^{\prime}+1}^{2}\left(\mathbb{Z}^{\prime}, \mathbb{C}^{4}\right)\right\}, \quad X^{\prime}=\left\{\left(\mathbf{c}^{+}, \mathbf{c}^{-}, \overline{\mathbf{c}}^{+}, \overline{\mathbf{c}}^{-}\right) \in l_{s^{\prime}}^{2}\left(\mathbb{Z}^{\prime}, \mathbb{C}^{4}\right)\right\},
$$

for $s^{\prime}=s-\frac{1}{4}$. (Here we have used the fact that the convolution sum acts on $\left\{\psi_{m}\right\}_{m \in \mathbb{Z}}$, where $\psi_{m}$ is given by $c_{m}^{ \pm}$in the transformation (3.10)-(3.11).) If the space $X$ is Banach algebra for $s>\frac{1}{2}$, the space $X^{\prime}$ is Banach algebra with respect to the decaying weights for $s^{\prime}>\frac{1}{4}$. The domain for reversible solutions become now

$$
D_{r}^{\prime}=\left\{\left(\mathbf{c}^{+}, \mathbf{c}^{-}, \overline{\mathbf{c}}^{+}, \overline{\mathbf{c}}^{-}\right) \in D^{\prime}: \quad c_{m}^{ \pm}(-y)=\bar{c}_{m}^{ \pm}(y), m \in \mathbb{Z}_{-}^{\prime}, \quad c_{m}^{ \pm}(-y)=\bar{c}_{m}^{\mp}(y), m \in \mathbb{Z}_{+}^{\prime},\right\}
$$

and the reversibility constraint becomes

$$
\Sigma_{r}^{\prime}=\left\{\left(\mathbf{c}^{+}, \mathbf{c}^{-}, \overline{\mathbf{c}}^{+}, \overline{\mathbf{c}}^{-}\right) \in D^{\prime}: \operatorname{Im} c_{m}^{ \pm}=0, m \in \mathbb{Z}_{-}^{\prime}, \quad \operatorname{Re} c_{m}^{+}=\operatorname{Re} c_{m}^{-}, \operatorname{Im} c_{m}^{+}=-\operatorname{Im} c_{m}^{-}, m \in \mathbb{Z}_{+}^{\prime}\right\} .
$$


In what follows, we are concerned with the reversible homoclinic orbit of the Hamilton's equations of motion (3.13) - (3.14) in normal coordinates (3.10)-(3.11). We will suppress the explicit dependence of $H$ from $\left(\overline{\mathbf{c}}^{+}, \overline{\mathbf{c}}^{-}\right)$for briefness of notations.

Remark 4 Since $k_{m}^{ \pm}>0$ for sufficiently large negative $m \in \mathbb{Z}_{-}^{\prime}$, the center manifold $E_{c}^{+} \oplus E_{c}^{-}$is spanned by an infinite set of modes with positive and negative energies. Therefore, we can not use the technique of [5] which relies on the fact that the quadratic part of the Hamiltonian function is positivedefinite for the non-bifurcating modes of center manifold. We can however use the technique of [6] which relies on the separation of slow motion for the modes, which correspond to the zero eigenvalue (modes $c_{n}^{+}$and $c_{-n}^{-}$), and the fast motion of the other modes, which correspond to non-zero purely imaginary or complex eigenvalues (modes $c_{m}^{ \pm}$for all other $m$ ). Moreover, we can simplify the technique of [6] by incorporating the Hamiltonian structure (3.13)-(3.14) with the Hamiltonian function (3.12).

\section{Normal form and persistence of homoclinic orbits}

We first show that the formal truncation of the Hamiltonian function (3.12) at the two bifurcating modes $c_{n}^{+}$and $c_{-n}^{-}$, which correspond to the double zero eigenvalue of the linearized system at the zero solution for $\epsilon=0$, leads to the coupled-mode system (1.5) for $\epsilon \neq 0$. Then, we derive an extended coupled-mode system for the modes $c_{n}^{+}$and $c_{-n}^{-}$by using near-identity transformations of the Hamiltonian function and prove persistence of a reversible homoclinic orbit in the extended coupled-mode system. We will assume from now on that $0<c<n$.

Let us consider the subspace of the phase space of the Hamiltonian system (3.13)-(3.14):

$$
S=\left\{c_{m}^{+}=0, \forall m \in \mathbb{Z}^{\prime} \backslash\{n\}, \quad c_{m}^{-}=0, \forall m \in \mathbb{Z}^{\prime} \backslash\{-n\}\right\} .
$$

Denote $Q=S^{\perp}$. If the Hamiltonian function $H$ is formally constrained on the subspace $S$, the expression (3.12) takes the form

$$
\left.H\right|_{S}=\epsilon\left[\frac{\Omega\left|c_{n}^{+}\right|^{2}}{n-c}+\frac{\Omega\left|c_{-n}^{-}\right|^{2}}{n+c}-\frac{w_{2 n}\left(\bar{c}_{n}^{+} c_{-n}^{-}+c_{n}^{+} \bar{c}_{-n}^{-}\right)}{\sqrt{n^{2}-c^{2}}}-\frac{\sigma}{2}\left(\frac{\left|c_{n}^{+}\right|^{4}}{(n-c)^{2}}+\frac{4\left|c_{n}^{+}\right|^{2}\left|c_{-n}^{-}\right|^{2}}{n^{2}-c^{2}}+\frac{\left|c_{-n}^{-}\right|^{4}}{(n+c)^{2}}\right)\right] .
$$

Since $(n,-n) \in \mathbb{Z}_{-}^{\prime}$, we use the symplectic structure (3.13) to generate a system of first-order ODEs for normal coordinates $c_{n}^{+}$and $c_{-n}^{-}$. By using the new independent variable $Y=\epsilon y$ and the new dependent variables $a=\frac{c_{n}^{+}}{\sqrt{n-c}}$ and $b=\frac{c_{-n}^{-}}{\sqrt{n+c}}$, we recover the ODE system

$$
\left\{\begin{array}{cl}
i(n-c) a^{\prime}+\Omega a & =w_{2 n} b+\sigma\left(|a|^{2}+2|b|^{2}\right) a \\
-i(n+c) b^{\prime}+\Omega b & =w_{2 n} a+\sigma\left(2|a|^{2}+|b|^{2}\right) b
\end{array}\right.
$$

where the derivatives are taken with respect to $Y=\epsilon y$. The system (4.2) is nothing but the coupledmode system (1.5) after the separation of the traveling variable $Y=X-c T$ and the stationary variable $T$ in the transformation

$$
a(X, T) \mapsto a(Y) e^{-i \Omega T}, \quad b(X, T) \mapsto b(Y) e^{-i \Omega T}, \quad Y=X-c T,
$$

with the correspondence $Y=\sqrt{n^{2}-c^{2}} \xi$ and $\Omega=\frac{\mu n\left(1-c^{2}\right)}{\sqrt{n^{2}-c^{2}}}$. The system (4.2) has a localized solution (a homoclinic orbit) (see Section 1) for $w_{2 n} \neq 0$ and $|\Omega|<\Omega_{0}$, where $\Omega_{0}=\left|w_{2 n}\right| \frac{\sqrt{n^{2}-c^{2}}}{n}$. 
The ODE system (4.2) is invariant with respect to translation $a(Y) \rightarrow a\left(Y-Y_{0}\right), b(Y) \rightarrow b\left(Y-Y_{0}\right)$ for all $Y_{0} \in \mathbb{R}$ and gauge transformation $a(Y) \rightarrow e^{i \alpha} a\left(Y-Y_{0}\right), b(Y) \rightarrow e^{i \alpha} b\left(Y-Y_{0}\right)$ for all $\alpha \in \mathbb{R}$. Therefore, any solution of the system is continued with a two-parameter group of symmetry transformations. However, these parameters are set uniquely in the reversible homoclinic orbit of Definition 11. In addition, we note that although the ODE system (4.2) is formulated in the four-dimensional phase space, it has two conserved quantities on $Y \in \mathbb{R}$ related to the translational and gauge symmetries. Indeed, the Hamiltonian $\left.H\right|_{S}$ and the quadratic function

$$
Q=\left|c_{n}^{+}\right|^{2}-\left|c_{-n}^{-}\right|^{2}=(n-c)|a|^{2}-(n+c)|b|^{2}
$$

are constants on $Y \in \mathbb{R}$. As a result, localized solutions of the system (4.2) are defined on a subspace which obeys a planar Hamiltonian system. This planar Hamiltonian system is given by the second equation of the system (1.10) in variables $(\phi, \bar{\phi})$. We recall that reversible homoclinic orbits of planar Hamiltonian systems are structurally stable with respect to parameter continuations.

To incorporate the ideas of integrability of the Hamiltonian coupled-mode system on the subspace $S$ and persistence of the reversible homoclinic orbit in the planar Hamiltonian system, we extend the coupled-mode system by using near-identity transformations and the normal form theory.

Lemma 4 Let $0<c<n$, such that $\frac{n^{2}+c^{2}}{2 c} \notin \mathbb{Z}^{\prime}$. For each $N \in \mathbb{N}$ and sufficiently small $\epsilon$, there is a near-identity, analytic, symplectic change of coordinates in a neighborhood of the origin in $X^{\prime}$ in (3.15), such that the Hamiltonian function $H$ in (3.12) transforms to the normal form up to the order of $\mathrm{O}\left(\epsilon^{N+1}\right)$,

$$
\begin{aligned}
H=\sum_{m \in \mathbb{Z}_{-}^{\prime}} & \left(k_{m}^{+}\left|c_{m}^{+}\right|^{2}-k_{m}^{-}\left|c_{m}^{-}\right|^{2}\right)+\sum_{m \in \mathbb{Z}_{+}^{\prime}}\left(\kappa_{m}^{-} c_{m}^{-} \bar{c}_{m}^{+}-\kappa_{m}^{+} c_{m}^{+} \bar{c}_{m}^{-}\right) \\
& +\epsilon H_{S}\left(c_{n}^{+}, c_{-n}^{-}\right)+\epsilon H_{Q}\left(c_{n}^{+}, c_{-n}^{-}, \mathbf{c}^{+}, \mathbf{c}^{-}\right)+\epsilon^{N+1} H_{R}\left(c_{n}^{+}, c_{-n}^{-}, \mathbf{c}^{+}, \mathbf{c}^{-}\right),
\end{aligned}
$$

where $H_{S}$ is a polynomial of the degree $2 N+2$ in $\left(c_{n}^{+}, c_{-n}^{-}\right)$on $S, H_{Q}$ is a polynomial of the degree $2 N$ in $\left(c_{n}^{+}, c_{-n}^{-}\right)$on $S$ and of the degree 4 in $\left(\mathbf{c}^{+}, \mathbf{c}^{-}\right)$on $Q$ with no linear terms with respect to $\left(\mathbf{c}^{+}, \mathbf{c}^{-}\right)$on $Q$, and $H_{R}$ is a polynomial of the degree $8 N+4$ in $\left(c_{n}^{+}, c_{-n}^{-}\right)$on $S$ and of the degree 4 in $\left(\mathbf{c}^{+}, \mathbf{c}^{-}\right)$on Q. All components $H_{S}, H_{Q}$ and $H_{R}$ depend on $\epsilon$, such that $H_{S}$ and $H_{Q}$ are polynomials in $\epsilon$ of the degree $N-1$ and $H_{R}$ is a polynomial in $\epsilon$ of the degree $3 N-1$. The reversibility (3.6) and gauge (3.7) transformations are preserved by the change of the variables.

Proof. The existence of a near-identity symplectic transformation that maps $H$ to the form (4.4) follows from the fact that the non-resonance conditions $l \kappa_{0}-\kappa_{m}^{ \pm} \neq 0$ are satisfied for any $l \in \mathbb{Z}$ and $m \in \mathbb{Z}^{\prime}$ since $\kappa_{0}=0$ and all eigenvalues are semi-simple for $\epsilon=0$. See [5] for an iterative sequence of symplectic transformations. The transformation is analytic in a local neighborhood of the origin in $X^{\prime}$ as the vector field of the Hamiltonian system (3.13)-(3.14) is analytic (given by a cubic polynomial). The reversibility (3.6) and gauge (3.7) symmetries are preserved by the symplectic change of variables [5]. The count of the degree of polynomials $H_{S}, H_{Q}$ and $H_{R}$ follows from the fact that the vector field of the Hamiltonian system (3.13)-(3.14) contains only linear and cubic terms in normal coordinates, while the near-identity transformation of $\left(\mathbf{c}^{+}, \mathbf{c}^{-}\right)$on $Q$ up to the order of $\mathrm{O}\left(\epsilon^{N+1}\right)$ involves a polynomial in $\epsilon$ of the degree $N$ and a polynomial in $\left(c_{n}^{+}, c_{-n}^{-}\right)$on $S$ of the degree $2 N+1$.

Example 2 For $N=1$, the near-identity transformation for $c_{m}^{+}, m \in \mathbb{Z}_{-}^{\prime} \backslash\{n\}$ takes the explicit form

$$
\tilde{c}_{m}^{+}=c_{m}^{+}-\frac{\epsilon}{k_{m}^{+}}\left[w_{m, n} c_{n}^{+}+w_{m,-n} c_{-n}^{-}+\sigma\left(g_{n, n, n}\left(c_{n}^{+}\right)^{2} \bar{c}_{-n}^{-} \delta_{m, 3 n}+g_{-n,-n,-n}\left|c_{n}^{+}\right|^{2} c_{-n}^{-} \delta_{m,-n}\right)\right]+\mathrm{O}\left(\epsilon^{2}\right),
$$


where $\tilde{c}_{m}^{+}$is a new variable. Similar explicit formulas can be obtained for $c_{m}^{-}, m \in \mathbb{Z}_{-}^{\prime} \backslash\{-n\}$ and for $c_{m}^{ \pm}, m \in \mathbb{Z}_{+}^{\prime}$. We note that the tilde signs are omitted for new variables in the expression (4.4).

Remark 5 For each $N \in \mathbb{N}$, the subspace $S$ defined by (4.1) is an invariant subspace of the Hamiltonian system (3.13) -(3.14) with the Hamiltonian function (4.4) truncated at $H_{R} \equiv 0$. The dynamics on $S$ is given by the four-dimensional Hamiltonian system

$$
\frac{d c_{n}^{+}}{d Y}=i \frac{\partial H_{S}}{\partial \bar{c}_{n}^{+}}, \quad \frac{d c_{-n}^{-}}{d Y}=-i \frac{\partial H_{S}}{\partial \bar{c}_{-n}^{+}}
$$

where $Y=\epsilon y$. If $N=1$, the system (4.5) transforms to the coupled-mode system (4.2) in variables $a=\frac{c_{n}^{+}}{\sqrt{n-c}}$ and $b=\frac{c_{-n}^{-}}{\sqrt{n+c}}$. If $N>1$, this system is referred to as the extended coupled-mode system.

Lemma 5 Let $w_{2 n} \neq 0$ for a given $n \in \mathbb{N}$. For each $N \in \mathbb{N}$ and sufficiently small $\epsilon$, there exists a reversible homoclinic orbit of the system (4.5) for $|\Omega|<\Omega_{0}=\left|w_{2 n}\right| \frac{\sqrt{n^{2}-c^{2}}}{n}$. Moreover, the solution for the homoclinic orbit satisfies the global bound

$$
\left|c_{n}^{+}(y)\right| \leq C_{+} e^{-\epsilon \gamma|y|}, \quad\left|c_{-n}^{-}(y)\right| \leq C_{-} e^{-\epsilon \gamma|y|}, \quad \forall y \in \mathbb{R}
$$

for some $\epsilon$-independent constants $\gamma>0$ and $C_{ \pm}>0$.

Proof. Due to the gauge-invariance of the polynomial Hamiltonian function $H_{S}=H_{S}\left(c_{n}^{+}, c_{-n}^{-}\right)$, it must satisfy the partial differential equation [2]:

$$
\left.\frac{d}{d \alpha} H_{S}\left(e^{i \alpha} c_{n}^{+}, e^{i \alpha} c_{-n}^{-}\right)\right|_{\alpha=0} \simeq c_{n}^{+} \frac{\partial H_{S}}{\partial c_{n}^{+}}-\bar{c}_{n}^{+} \frac{\partial H_{S}}{\partial \bar{c}_{n}^{+}}+c_{-n}^{-} \frac{\partial H_{S}}{\partial c_{-n}^{-}}-\bar{c}_{-n}^{-} \frac{\partial H_{S}}{\partial c_{-n}^{-}}=0 .
$$

It follows from the system (4.5) and the relation (4.7) that $Q=\left|c_{n}^{+}\right|^{2}-\left|c_{-n}^{-}\right|^{2}$ is constant in $Y \in \mathbb{R}[2]$. If localized solutions exist, then $Q=0$. Let us represent the solutions in the general form

$$
c_{n}^{+}=\sqrt{\rho+q} e^{i \varphi+i \theta}, \quad c_{-n}^{-}=\sqrt{\rho-q} e^{i \varphi-i \theta},
$$

where $(\rho, q, \theta, \varphi)$ are new real-valued variables. Using the chain rule for $H_{S}=\tilde{H}_{S}(\rho, q, \theta, \varphi)$, we find that $\varphi$ is a cyclic variable of the Hamiltonian function $\tilde{H}_{S}$ and $q$ is constant due to the gauge invariance (4.7). Setting $q=0$, we find that $(\rho, \theta)$ satisfy a planar Hamiltonian system, while $\varphi$ is found from a linear inhomogeneous equation:

$$
2 \frac{d \rho}{d Y}=-\left.\frac{\partial \tilde{H}_{S}}{\partial \theta}\right|_{q=0}, \quad 2 \frac{d \theta}{d Y}=\left.\frac{\partial \tilde{H}_{S}}{\partial \rho}\right|_{q=0}, \quad 2 \frac{d \varphi}{d Y}=\left.\frac{\partial \tilde{H}_{S}}{\partial q}\right|_{q=0}
$$

where $\left.\tilde{H}_{S}\right|_{q=0}$ is a function of $(\rho, \theta)$. If $\epsilon=0$, the system (4.9) reduces to the ODE system (1.10) rewritten in new coordinates and for $\phi=\sqrt{\rho} e^{i \theta}$. The vector field of the extended coupled-mode system (4.5) is given by polynomials in $c_{n}^{+}$and $c_{-n}^{-}$of the degree $2 N+1$ and in $\epsilon$ of the degree $N-1$. Recall that the coupled-mode system (4.2) admits a reversible homoclinic orbit for $w_{2 n} \neq 0$ and $|\Omega|<\Omega_{0}$, where $\Omega_{0}=\left|w_{2 n}\right| \frac{\sqrt{n^{2}-c^{2}}}{n}$. Since a reversible homoclinic orbit is structurally stable in a planar Hamiltonian system with an analytic vector field, the homoclinic orbit persists in the extended coupled-mode system (4.5) for sufficiently small $\epsilon$. 


\section{Construction of local center and center-stable manifolds}

We study solutions of the Hamiltonian system of equations (3.13)-(3.14) after the normal-form transformation of Lemma 4. We construct a local solution on $y \in\left[0, L / \epsilon^{N+1}\right]$ for some $\epsilon$-independent constant $L>0$, which is close to the homoclinic orbit of Lemma 5 by the distance $C \epsilon^{N}$ for some $\epsilon$-independent constant $C>0$ in vector norm on $X^{\prime}$. This solution represents an infinite-dimensional local centerstable manifold and it is spanned by the small bounded oscillatory and small exponentially decaying solutions near the exponentially decaying homoclinic solution with the decay bound (4.6). Parameters of the local center-stable manifold are chosen to ensure that the manifold intersects at $y=0$ with the symmetric section $\Sigma_{r}^{\prime}$. This construction completes the proof of the main Theorem 2 .

By using Lemma 4 and the explicit representation of the Hamiltonian function (4.4), we rewrite the Hamiltonian system of equations in the separated form

$$
\begin{aligned}
\frac{d c_{n}^{+}}{d y} & =\epsilon\left[\mu_{\epsilon}^{+} c_{n}^{+}+\nu_{\epsilon}^{+} c_{-n}^{-}+F_{S}^{+}\left(c_{n}^{+}, c_{-n}^{-}\right)+F_{Q}^{+}\left(c_{n}^{+}, c_{-n}^{-}, \mathbf{c}\right)\right]+\epsilon^{N+1} F_{R}^{+}\left(c_{n}^{+}, c_{n}^{-}, \mathbf{c}\right), \\
\frac{d c_{-n}^{-}}{d y} & =\epsilon\left[\mu_{\epsilon}^{-} c_{n}^{+}+\nu_{\epsilon}^{-} c_{-n}^{-}+F_{S}^{-}\left(c_{n}^{+}, c_{-n}^{-}\right)+F_{Q}^{-}\left(c_{n}^{+}, c_{-n}^{-}, \mathbf{c}\right)\right]+\epsilon^{N+1} F_{R}^{-}\left(c_{n}^{+}, c_{n}^{-}, \mathbf{c}\right), \\
\frac{d \mathbf{c}}{d y} & =\Lambda_{\epsilon} \mathbf{c}+\epsilon \mathbf{F}_{Q}\left(c_{n}^{+}, c_{-n}^{-}, \mathbf{c}\right)+\epsilon^{N+1} \mathbf{F}_{R}\left(c_{n}^{+}, c_{n}^{-}, \mathbf{c}\right),
\end{aligned}
$$

where $\mathbf{c}$ denotes all components of $\left(\mathbf{c}^{+}, \mathbf{c}^{-}, \overline{\mathbf{c}}^{+}, \overline{\mathbf{c}}^{-}\right)$in $Q=S^{\perp},\left(\mu_{\epsilon}^{ \pm}, \nu_{\epsilon}^{ \pm}, \Lambda_{\epsilon}\right)$ denote the coefficient matrix for the linear part of the system and $\left(F_{S}^{ \pm}, F_{Q}^{ \pm}, F_{R}^{ \pm}, \mathbf{F}_{Q}, \mathbf{F}_{R}\right)$ denote the nonlinear (polynomial) part of the system. For briefness of notations, we do not rewrite the subsystem (5.1)-(5.2) for variables $\left(\bar{c}_{n}^{+}, \bar{c}_{-n}^{-}\right)$and we do not write dependence of the nonlinear functions from these variables. The variables c are equivalent to tilde-variables in Example 2 after the near-identity transformations, but the tildenotations are dropped for simplicity of notations.

Lemma 6 Let $W(x)$ satisfy Assumption 1 and $w_{2 n} \neq 0$ for a given $n \in \mathbb{N}$. For sufficiently small $\epsilon$, the linearized system (5.1)-(5.3) at the zero solution for $|\Omega|<\Omega_{0}=\left|w_{2 n}\right| \frac{\sqrt{n^{2}-c^{2}}}{n}$ and $0<c<n$ is topologically equivalent to the one for $\epsilon=0$, except that the double zero eigenvalue of the subsystem (5.1)-(5.2) splits into a pair of complex eigenvalues to the left and right half-planes.

Proof. Since all non-zero eigenvalues of the linearized Hamiltonian system (5.3) at the zero solution are semi-simple at $\epsilon=0$, they are structurally stable in the perturbation theory for sufficiently small $\epsilon \neq 0$. The matrix operator $\Lambda_{\epsilon}$ is a polynomial in $\epsilon$ and $\Lambda_{0}$ is a diagonal unbounded matrix operator which consists of $\left(i k_{m}^{+},-i k_{m}^{-},-i k_{m}^{+}, i k_{m}^{-}\right)$for $m \in \mathbb{Z}_{-}^{\prime}$ and of $\left(\kappa_{m}^{+}, \kappa_{m}^{-}, \bar{\kappa}_{m}^{+}, \bar{\kappa}_{m}^{-}\right)$for $m \in \mathbb{Z}_{+}^{\prime}$. The matrix operator with elements $\epsilon w_{m, m^{\prime}}$ represents a small perturbation to $\Lambda_{0}$ if the vector of Fourier coefficients $\mathbf{W}$ is in $l^{1}(\mathbb{Z}) \subset l_{s}^{2}(\mathbb{Z})$ for $s>\frac{1}{2}$ according to Assumption 1 .

The coefficients $\mu_{\epsilon}^{ \pm}$and $\nu_{\epsilon}^{ \pm}$are polynomials in $\epsilon$ and

$$
\mu_{0}^{+}=\frac{i \Omega}{n-c}, \quad \mu_{0}^{-}=\frac{i w_{2 n}}{\sqrt{n^{2}-c^{2}}}, \quad \nu_{0}^{+}=\frac{-i w_{2 n}}{\sqrt{n^{2}-c^{2}}}, \quad \nu_{0}^{-}=\frac{-i \Omega}{n+c} .
$$

At $\epsilon=0$, the linearized subsystem (5.1)-(5.2) corresponds to the linearized coupled-mode system (4.2). Its characteristic equation is given by

$$
\left(n^{2}-c^{2}\right) \kappa^{2}-2 i \epsilon c \Omega \kappa+\epsilon^{2}\left(\Omega^{2}-w_{2 n}^{2}\right)=0,
$$


with two roots

$$
\kappa=\kappa_{ \pm}=\epsilon \frac{i \Omega c \pm \sqrt{\left(n^{2}-c^{2}\right) w_{2 n}^{2}-n^{2} \Omega^{2}}}{n^{2}-c^{2}} .
$$

The two roots have $\operatorname{Re} \kappa_{ \pm} \gtrless 0$ if $|\Omega|<\Omega_{0}$, where $\Omega_{0}=\left|w_{2 n}\right| \frac{\sqrt{n^{2}-c^{2}}}{n}$. Under the same assumption on $\mathbf{W}$, perturbation terms in $\mu_{\epsilon}^{ \pm}$and $\nu_{\epsilon}^{ \pm}$are small compared to the leading-order terms $\mu_{0}^{ \pm}$and $\nu_{0}^{ \pm}$, such that the pair persists in the left half-plane and right half-plane of the $\kappa$-plane.

Corollary 1 For sufficiently small $\epsilon$, a local neighborhood of the zero point in the phase space $X^{\prime}$ can be decomposed into the subspaces determined by the spectrum of the linearized system at the zero solution

$$
X^{\prime}=X_{h} \oplus X_{c} \oplus X_{u} \oplus X_{s},
$$

where $X_{s}$ and $X_{u}$ are associated to the subspaces $E^{s}$ and $E^{u}$ of Lemma 1, while $X_{h}$ and $X_{c}$ are associated to the subspaces $E^{c^{+}} \oplus E^{c^{-}}$on $S$ and $Q$ respectively.

By Remark 5, the truncated system (5.1)-(5.3) with $F_{R}^{ \pm} \equiv 0$ and $\mathbf{F}_{R} \equiv \mathbf{0}$ admits an invariant reduction on $S$. By Lemma 5, the extended coupled-mode system (4.5) on $S$ has a reversible homoclinic orbit which satisfies the decay bound (4.6). This construction enables us to represent the solution of the subsystem (5.1)-(5.2) and its complex conjugate in the form $\left[c_{n}^{+}, c_{-n}^{-}, \bar{c}_{n}^{+}, \bar{c}_{-n}^{-}\right]^{T}=\mathbf{c}_{0}(\epsilon y)+\mathbf{c}_{h}(y)$, where $\mathbf{c}_{0}(\epsilon y)$ is the homoclinic orbit of Lemma 5 and $\mathbf{c}_{h}(y)$ is a perturbation term. By using the decomposition, we rewrite the system (5.1)-(5.3) in the equivalent form

$$
\begin{aligned}
\frac{d \mathbf{c}_{h}}{d y} & =\epsilon \Lambda_{h}\left(\mathbf{c}_{0}\right) \mathbf{c}_{h}+\epsilon \mathbf{G}_{Q}\left(\mathbf{c}_{0}\right)\left(\mathbf{c}_{h}, \mathbf{c}\right)+\epsilon^{N+1} \mathbf{G}_{R}\left(\mathbf{c}_{0}+\mathbf{c}_{h}, \mathbf{c}\right), \\
\frac{d \mathbf{c}}{d y} & =\Lambda_{\epsilon} \mathbf{c}+\epsilon \mathbf{F}_{Q}\left(\mathbf{c}_{0}+\mathbf{c}_{h}, \mathbf{c}\right)+\epsilon^{N+1} \mathbf{F}_{R}\left(\mathbf{c}_{0}+\mathbf{c}_{h}, \mathbf{c}\right),
\end{aligned}
$$

where $\Lambda_{h}\left(\mathbf{c}_{0}\right)$ is a 4-by-4 linearization matrix of the extended coupled-mode system (4.5) and its complex conjugate at the solution $\mathbf{c}_{0}(\epsilon y)$ and $\left(\mathbf{G}_{Q}, \mathbf{G}_{R}\right)$ denote the nonlinear part of the subsystem (5.1)-(5.2) and its conjugate. We note that the function $\mathbf{G}_{Q}$ combines nonlinear terms in $\mathbf{c}_{h}$ from the functions $F_{S}^{ \pm}$ and the nonlinear terms in $\mathbf{c}$ from the functions $F_{Q}^{ \pm}$. For simplicity of notations, we say that $\mathbf{c}_{h} \in X_{h}$ and $\mathbf{c} \in X_{h}^{\perp}$ in the decomposition $X^{\prime}=X_{h} \oplus X_{h}^{\perp}$.

Example 3 For $N=1$, the linearization matrix $\Lambda_{h}\left(\mathbf{c}_{0}\right)$ takes the explicit form

$$
i\left[\begin{array}{cccc}
\frac{\Omega}{n-c}-\frac{2 \sigma\left|c_{n}^{+}\right|^{2}}{(n-c)^{2}}-\frac{2 \sigma\left|c_{-n}^{-}\right|^{2}}{n^{2}-c^{2}} & -\frac{w_{2 n}}{\sqrt{n^{2}-c^{2}}}-\frac{2 \sigma c_{n}^{+} \bar{c}_{-n}^{-}}{n^{2}-c^{2}} & -\frac{\sigma c_{n}^{+2}}{(n-c)^{2}} & -\frac{2 \sigma c_{n}^{+} c_{-n}^{-}}{n^{2}-c^{2}} \\
\frac{w_{2 n}}{\sqrt{n^{2}-c^{2}}}+\frac{2 \sigma \bar{c}_{n}^{+} c_{-n}^{-}}{n^{2}-c^{2}} & -\frac{\Omega}{n+c}+\frac{2 \sigma\left|c_{n}^{+}\right|^{2}}{n^{2}-c^{2}}+\frac{2 \sigma\left|c_{-n}^{-}\right|^{2}}{(n+c)^{2}} & \frac{2 \sigma c_{n}^{+} c_{-n}^{-}}{n^{2}-c^{2}} & \frac{\sigma c_{-n}^{-2}}{(n+c)^{2}} \\
\frac{\sigma \bar{c}_{n}^{+2}}{(n-c)^{2}} & \frac{2 \sigma \bar{c}_{n}^{+} \bar{c}_{-n}^{-}}{n^{2}-c^{2}} & -\frac{\Omega}{n-c}+\frac{2 \sigma\left|c_{n}^{+}\right|^{2}}{(n-c)^{2}}+\frac{2 \sigma\left|c_{-n}^{-}\right|^{2}}{n^{2}-c^{2}} & \frac{w_{2 n}}{\sqrt{n^{2}-c^{2}}}+\frac{2 \sigma \bar{c}_{n}^{+} c_{-n}^{-}}{n^{2}-c^{2}} \\
-\frac{2 \sigma \bar{c}_{n}^{+} \bar{c}_{-n}^{-}}{n^{2}-c^{2}} & -\frac{\sigma \bar{c}_{-n}^{-2}}{(n+c)^{2}} & -\frac{w_{2 n}}{\sqrt{n^{2}-c^{2}}}-\frac{2 \sigma c_{n}^{+} \bar{c}_{-n}^{-}}{n^{2}-c^{2}} & \frac{\Omega}{n+c}-\frac{2 \sigma\left|c_{n}^{+}\right|^{2}}{n^{2}-c^{2}}-\frac{2 \sigma\left|c_{-n}^{-}\right|^{2}}{(n+c)^{2}}
\end{array}\right],
$$

where components of $\left[c_{n}^{+}, c_{-n}^{-}, \bar{c}_{n}^{+}, \bar{c}_{-n}^{-}\right]^{T}$ are evaluated at the solution $\mathbf{c}_{0}$ of the extended coupled-mode equation (4.5). As $|y| \rightarrow \infty$, the matrix $\Lambda_{h}\left(\mathbf{c}_{0}\right)$ converges to the form

$$
\Lambda_{h}(\mathbf{0})=i\left[\begin{array}{cccc}
\frac{\Omega}{n-c} & -\frac{w_{2 n}}{\sqrt{n^{2}-c^{2}}} & 0 & 0 \\
\frac{w_{2 n}}{\sqrt{n^{2}-c^{2}}} & -\frac{\Omega}{n+c} & 0 & 0 \\
0 & 0 & -\frac{\Omega}{n-c} & \frac{w_{2 n}}{\sqrt{n^{2}-c^{2}}} \\
0 & 0 & -\frac{w_{2 n}}{\sqrt{n^{2}-c^{2}}} & \frac{\Omega}{n+c}
\end{array}\right]
$$


such that $\left\|\Lambda_{h}\left(\mathbf{c}_{0}\right)-\Lambda_{h}(\mathbf{0})\right\|_{X_{h} \mapsto X_{h}} \leq C e^{-\epsilon \gamma|y|}$ for some $C>0$ and $\gamma>0$ according to the decay bound (4.6).

Lemma 7 Let $w_{2 n} \neq 0$ for a given $n \in \mathbb{N},|\Omega|<\Omega_{0}=\left|w_{2 n}\right| \frac{\sqrt{n^{2}-c^{2}}}{n}$, and $0<c<n$. Consider the linear inhomogeneous equation

$$
\frac{d \mathbf{c}_{h}}{d y}-\epsilon \Lambda_{h}\left(\mathbf{c}_{0}\right) \mathbf{c}_{h}=\mathbf{F}_{h}(y)
$$

where $\mathbf{F}_{h} \in C_{b}^{0}(\mathbb{R})$. The homogeneous equation has a two-dimensional stable manifold spanned by the two fundamental solutions

$$
\mathbf{s}_{1}=\mathbf{c}_{0}^{\prime}(y), \quad \mathbf{s}_{2}=\sigma_{1} \mathbf{c}_{0}(y),
$$

where $\sigma_{1}$ is a diagonal matrix of $(1,1,-1,-1)$. If components of $\mathbf{F}_{h}(y)$ satisfies the constraints $\forall y \in \mathbb{R}$

$$
\left(\mathbf{F}_{h}\right)_{1}(y)=\left(\overline{\mathbf{F}}_{h}\right)_{1}(-y), \quad\left(\mathbf{F}_{h}\right)_{2}(y)=\left(\overline{\mathbf{F}}_{h}\right)_{2}(-y), \quad\left(\mathbf{F}_{h}\right)_{3}(y)=-\left(\overline{\mathbf{F}}_{h}\right)_{1}(y), \quad\left(\mathbf{F}_{h}\right)_{4}(y)=-\left(\overline{\mathbf{F}}_{h}\right)_{4}(y),
$$

then there exists a two-parameter family of solutions $\mathbf{c}_{h} \in C_{b}^{0}(\mathbb{R})$ in the form $\mathbf{c}_{h}=\alpha_{1} \mathbf{s}_{1}(y)+\alpha_{2} \mathbf{s}_{2}(y)+$ $\tilde{\mathbf{c}}_{h}(y)$, where $\left(\alpha_{1}, \alpha_{2}\right)$ are parameters and $\tilde{\mathbf{c}}_{h}(y)$ is a particular solution of the inhomogeneous equation (5.7), such that $\left\|\tilde{\mathbf{c}}_{h}\right\|_{C_{b}^{0}(\mathbb{R})} \leq \frac{C}{\epsilon}\left\|\mathbf{F}_{h}\right\|_{C_{b}^{0}(\mathbb{R})}$ for an $\epsilon$-independent constant $C$.

Proof. The existence of the two-dimensional kernel (5.8) follows from symmetries of the extended coupled-mode system (4.5) with respect to translation and gauge transformation. Since the subspace $X_{h}$ associated with $\Lambda_{h}(\mathbf{0})$ is invariant under $\Lambda_{h}\left(\mathbf{c}_{0}\right)$, the kernel is exactly two-dimensional and the other two fundamental solutions of the homogeneous equation are exponentially growing. The adjoint homogeneous equation $\frac{d \mathbf{u}}{d y}=-\epsilon \Lambda_{h}^{*}\left(\mathbf{c}_{0}\right) \mathbf{u}$ has also a two-dimensional stable manifold spanned by the two fundamental solutions

$$
\mathbf{s}_{1}^{*}=\sigma_{2} \mathbf{c}_{0}^{\prime}(y), \quad \mathbf{s}_{2}^{*}=\sigma_{3} \mathbf{c}_{0}(y),
$$

where $\sigma_{2}$ and $\sigma_{3}$ are diagonal matrices of $(1,-1,-1,1)$ and $(1,-1,1,-1)$ respectively. Unless the vector function $\mathbf{F}_{h}$ is orthogonal to $\left\{\mathbf{s}_{1}^{*}, \mathbf{s}_{2}^{*}\right\}$, a solution of the linear inhomogeneous equation (5.7) grows exponentially as $|y| \rightarrow \infty$. However, $\left(\mathbf{s}_{1}^{*}, \mathbf{F}_{h}\right)=\left(\mathbf{s}_{2}^{*}, \mathbf{F}_{h}\right)=0$ if the constraints (5.9) are satisfied. By the Fredholm theory, $\mathbf{F}_{h}$ is in the range of the linear unbounded operator $\frac{d}{d y}-\epsilon \Lambda_{h}\left(\mathbf{c}_{0}\right)$, such that there exists a solution $\tilde{\mathbf{c}}_{h} \in C_{b}^{0}(\mathbb{R})$ of the inhomogeneous equation (5.7) such that $\left\|\tilde{\mathbf{c}}_{h}\right\|_{C_{b}^{0}(\mathbb{R})} \leq \frac{C}{\epsilon}\left\|\mathbf{F}_{h}\right\|_{C_{b}^{0}(\mathbb{R})}$ for an $\epsilon$-independent constant $C$. A general solution of the inhomogeneous problem has the form $\mathbf{c}_{h}=\alpha_{1} \mathbf{s}_{1}(y)+\alpha_{2} \mathbf{s}_{2}(y)+\tilde{\mathbf{c}}_{h}(y)$, where $\left(\alpha_{1}, \alpha_{2}\right)$ are parameters.

Lemma 8 The nonlinear part of the vector field of the system (5.5)-(5.6) is bounded in a local neighborhood of the zero point in $X^{\prime}=X_{h} \oplus X_{h}^{\perp}$ by

$$
\begin{gathered}
\left\|\mathbf{G}_{R}\right\|_{X_{h}} \leq N_{R}\left(\left\|\mathbf{c}_{0}+\mathbf{c}_{h}\right\|_{X_{h}}+\|\mathbf{c}\|_{X_{h}^{\perp}}\right), \quad\left\|\mathbf{F}_{R}\right\|_{X_{h}^{\perp}} \leq M_{R}\left(\left\|\mathbf{c}_{0}+\mathbf{c}_{h}\right\|_{X_{h}}+\|\mathbf{c}\|_{X_{h}^{\perp}}\right), \\
\left\|\mathbf{G}_{Q}\right\|_{X_{h}} \leq N_{Q}\left(\left\|\mathbf{c}_{h}\right\|_{X_{h}}^{2}+\|\mathbf{c}\|_{X_{h}^{\perp}}^{2}\right), \quad\left\|\mathbf{F}_{Q}\right\|_{X^{\prime}} \leq M_{Q}\left(\left\|\mathbf{c}_{0}+\mathbf{c}_{h}\right\|_{X_{h}}+\|\mathbf{c}\|_{X_{h}^{\perp}}\right)\|\mathbf{c}\|_{X_{h}^{\perp}},
\end{gathered}
$$

for some $N_{R}, M_{R}, N_{Q}, M_{Q}>0$.

Proof. The system (5.5)-(5.6) is semi-linear with polynomial vector field for a finite $N \in \mathbb{N}$ defined on the domain $D^{\prime} \subset X^{\prime}$, where $D^{\prime}$ and $X^{\prime}$ are given in (3.15). If $X$ is the Banach algebra for $s>\frac{1}{2}$, then $X^{\prime}$ is Banach algebra for $s^{\prime}>\frac{1}{4}$. The derivation of estimates (5.11)-(5.12) follows similarly to Lemmas 2 and 3 in [5]. The characterization of $\mathbf{G}_{Q}$ and $\mathbf{F}_{Q}$ is based on the fact that the Hamiltonian function $H_{Q}$ is quadratic with respect to $\mathbf{c}$ by Lemma 4 . 
Remark 6 Using Corollary 1, we denote $\Lambda_{c}=\left.\Lambda_{\epsilon}\right|_{X_{c}}, \Lambda_{u}=\left.\Lambda_{\epsilon}\right|_{X_{u}}, \Lambda_{s}=\left.\Lambda_{\epsilon}\right|_{X_{s}}$ for a block-diagonal decomposition of $\Lambda_{\epsilon}$ on the invariant subspaces $X_{c}, X_{u}$ and $X_{s}$ respectively. We also denote the coordinates of the decomposition by $\mathbf{c}=\left[\mathbf{c}_{c}, \mathbf{c}_{u}, \mathbf{c}_{s}\right]$ and the projection operators by $P_{c}, P_{u}, P_{s}$ respectively.

Theorem 3 (Local center-stable manifold) Let $\mathbf{a} \in X_{c}, \mathbf{b} \in X_{s}$ and $\left(\alpha_{1}, \alpha_{2}\right) \in \mathbb{C}^{2}$ be small such that

$$
\|\mathbf{a}\|_{X_{c}} \leq C_{a} \epsilon^{N}, \quad\|\mathbf{b}\|_{X_{s}} \leq C_{b} \epsilon^{N}, \quad\left|\alpha_{1}\right|+\left|\alpha_{2}\right| \leq C_{\alpha} \epsilon^{N} .
$$

for some $\epsilon$-independent constants $C_{a}, C_{b}, C_{\alpha}>0$. Under the conditions of Lemma [6, there exists a family $\mathbf{c}_{h}\left(y ; \mathbf{a}, \mathbf{b}, \alpha_{1}, \alpha_{2}\right)$ and $\mathbf{c}\left(y ; \mathbf{a}, \mathbf{b}, \alpha_{1}, \alpha_{2}\right)$ of local solutions of the system (5.5)-(5.6) such that $\mathbf{c}_{c}(0)=\mathbf{a}, \mathbf{c}_{s}=e^{y \Lambda_{s}} \mathbf{b}+\tilde{\mathbf{c}}_{s}(y), \mathbf{c}_{h}=\alpha_{1} \mathbf{s}_{1}(y)+\alpha_{2} \mathbf{s}_{2}(y)+\tilde{\mathbf{c}}_{h}(y)$ with uniquely defined $\tilde{\mathbf{c}}_{s}(y)$ and $\tilde{\mathbf{c}}_{h}(y)$, and the local solutions satisfy the bound

$$
\sup _{y \in\left[0, L / \epsilon^{N+1}\right]}\left\|\mathbf{c}_{h}(y)\right\|_{X_{h}} \leq C_{h} \epsilon^{N}, \quad \sup _{y \in\left[0, L / \epsilon^{N+1}\right]}\|\mathbf{c}(y)\|_{X_{h}^{\perp}} \leq C \epsilon^{N},
$$

for some $\epsilon$-independent constants $L>0$ and $C_{h}, C>0$.

Proof. We modify the system (5.5) -(5.6) by the following trick. We multiply the nonlinear vector field of the subsystem (5.6) by the cut-off function $\chi_{\left[0, y_{0}\right]}(y)$, such that

$$
\frac{d \mathbf{c}}{d y}=\Lambda_{\epsilon} \mathbf{c}+\epsilon \chi_{\left[0, y_{0}\right]}(y) \mathbf{F}_{Q}\left(\mathbf{c}_{0}+\mathbf{c}_{h}, \mathbf{c}\right)+\epsilon^{N+1} \chi_{\left[0, y_{0}\right]}(y) \mathbf{F}_{R}\left(\mathbf{c}_{0}+\mathbf{c}_{h}, \mathbf{c}\right),
$$

where $\chi_{\left[0, y_{0}\right]}(y)=1$ for $y \in\left[0, y_{0}\right] \subset \mathbb{R}$ for some $y_{0}>0$ and $\chi_{\left[0, y_{0}\right]}(y)=0$ otherwise. Similarly, we multiply the nonlinear vector field of the subsystem (5.5) by the cut-off function $\chi_{\left[0, y_{0}\right]}(y)$ and add symmetrically reflected vector field multiplied by the cut-off function $\chi_{\left[-y_{0}, 0\right]}(y)$, such that

$$
\begin{aligned}
\frac{d \mathbf{c}_{h}}{d y}-\epsilon \Lambda_{h}\left(\mathbf{c}_{0}\right) \mathbf{c}_{h}= & \epsilon \chi_{\left[0, y_{0}\right]}(y) \mathbf{G}_{Q}\left(\mathbf{c}_{0}\right)\left(\mathbf{c}_{h}, \mathbf{c}\right)+\epsilon^{N+1} \chi_{\left[0, y_{0}\right]}(y) \mathbf{G}_{R}\left(\mathbf{c}_{0}+\mathbf{c}_{h}, \mathbf{c}\right) \\
& \epsilon \chi_{\left[-y_{0}, 0\right]}(y) \mathbf{G}_{Q}^{*}\left(\mathbf{c}_{0}\right)\left(\mathbf{c}_{h}, \mathbf{c}\right)+\epsilon^{N+1} \chi_{\left[-y_{0}, 0\right]}(y) \mathbf{G}_{R}^{*}\left(\mathbf{c}_{0}+\mathbf{c}_{h}, \mathbf{c}\right),
\end{aligned}
$$

where $\left(\mathbf{G}_{Q, R}^{*}\right)_{1,2}(y)=\left(\overline{\mathbf{G}}_{Q, R}\right)_{1,2}(-y)$ and $\left(\mathbf{G}_{Q, R}^{*}\right)_{3,4}(y)=-\left(\overline{\mathbf{G}}_{Q, R}^{*}\right)_{1,2}(y)$ on $y \in\left[-y_{0}, 0\right)$. We are looking for a global solution of the system (5.15) -(5.16) in the space of bounded continuous functions $C_{b}^{0}(\mathbb{R})$. This global solution on $y \in \mathbb{R}$ corresponds to a local solution of the system (5.5)-(5.6) on the interval $y \in\left[0, y_{0}\right] \subset \mathbb{R}$.

The imaginary axis lies in the resolvent set of $\Lambda_{u}$ and $\Lambda_{s}$ and

$$
\left\|\left(\Lambda_{u, s}-i k I\right)^{-1}\right\|_{X_{u, s} \mapsto X_{u, s}} \leq \frac{K_{0}}{1+|k|}, \quad \forall k \in \mathbb{R},
$$

for some $K_{0}>0$. Let $\mathbf{c}_{s}(y)=e^{y \Lambda_{s}} \mathbf{b}+\tilde{\mathbf{c}}_{s}(y)$ and look for solution $\tilde{\mathbf{c}}_{s}(y)$ and $\mathbf{c}_{u}(y)$ of the system (5.15) projected to $X_{s}$ and $X_{u}$ with operators $P_{s}$ and $P_{u}$. By Lemmas 3, 8, and the Implicit Function Theorem, there exists a unique map from $C_{b}^{0}\left(\mathbb{R}, X_{h} \oplus X_{c}\right)$ to $C_{b}^{0}\left(\mathbb{R}, X_{u} \oplus X_{s}\right)$ parameterized by buch that

$$
\begin{aligned}
\sup _{y \in\left[0, y_{0}\right]}\left[\left\|\mathbf{c}_{u}(y)\right\|_{X_{u}}+\left\|\mathbf{c}_{s}(y)\right\|_{X_{s}}\right] \leq & \|\mathbf{b}\| X_{s}+\epsilon M_{1} \sup _{y \in\left[0, y_{0}\right]}\left[\left(1+\left\|\mathbf{c}_{h}(y)\right\|_{X_{h}}+\left\|\mathbf{c}_{c}(y)\right\| X_{X_{c}}\right)\left\|\mathbf{c}_{c}(y)\right\|_{X_{c}}\right] \\
& +\epsilon^{N+1} M_{2} \sup _{y \in\left[0, y_{0}\right]}\left[1+\left\|\mathbf{c}_{h}(y)\right\|_{X_{h}}+\left\|\mathbf{c}_{c}(y)\right\|_{X_{c}}\right]
\end{aligned}
$$


for some $M_{1}, M_{2}>0$.

Let $\mathbf{c}_{h}(y)=\alpha_{1} \mathbf{s}_{1}(y)+\alpha_{2} \mathbf{s}_{2}(y)+\tilde{\mathbf{c}}_{h}(y)$ and look for solution $\tilde{\mathbf{c}}_{h}(y)$ of the system (5.16). By the Hamiltonian structure of the system (5.5), the vector field satisfies the constraints $\left(\mathbf{G}_{Q, R}\right)_{3,4}(y)=$ $-\left(\overline{\mathbf{G}}_{Q, R}\right)_{1,2}(y)$ on $y \in\left[0, y_{0}\right]$. By the construction of the modified vector field, it satisfies the constraint (5.9). By Lemmas 7) 8, the bound (5.18), and the Implicit Function Theorem, there exists a unique map from $C_{b}^{0}\left(\mathbb{R}, X_{c}\right)$ to $C_{b}^{0}\left(\mathbb{R}, X_{h}\right)$ parameterized by $\left(\alpha_{1}, \alpha_{2}\right)$ and $\mathbf{b}$ such that

$$
\sup _{y \in\left[0, y_{0}\right]}\left\|\mathbf{c}_{h}(y)\right\|_{X_{h}} \leq\left|\alpha_{1}\right|+\left|\alpha_{2}\right|+M_{3} \sup _{y \in\left[0, y_{0}\right]}\left\|\mathbf{c}_{c}(y)\right\|_{X_{c}}^{2}+\epsilon^{N} M_{4} \sup _{y \in\left[0, y_{0}\right]}\left[1+\left\|\mathbf{c}_{c}(y)\right\|_{X_{c}}\right],
$$

for some $M_{3}, M_{4}>0$.

Since the spectrum of $\Lambda_{c}$ consists of pairs of semi-simple purely imaginary eigenvalues, the operator $\Lambda_{c}$ generates a strongly continuous group $e^{y \Lambda_{c}}$ for any $y \in \mathbb{R}$ on $X_{c}$ such that

$$
\sup _{y \in \mathbb{R}}\left\|e^{y \Lambda_{c}}\right\|_{X_{c} \mapsto X_{c}} \leq K,
$$

for some $K>0$. By variation of constant formula, the solution of the system (5.15) projected to $X_{c}$ can be rewritten in the integral form

$$
\mathbf{c}_{c}(y)=e^{y \Lambda_{c}} \mathbf{a}+\epsilon \int_{0}^{y} e^{\left(y-y^{\prime}\right) \Lambda_{c}} P_{c}\left[\mathbf{F}_{Q}\left(\mathbf{c}_{0}\left(\epsilon y^{\prime}\right)+\mathbf{c}_{h}\left(y^{\prime}\right), \mathbf{c}\left(y^{\prime}\right)\right)+\epsilon^{N} \mathbf{F}_{R}\left(\mathbf{c}_{0}\left(\epsilon y^{\prime}\right)+\mathbf{c}_{h}\left(y^{\prime}\right), \mathbf{c}\left(y^{\prime}\right)\right)\right] d y^{\prime},
$$

where $\mathbf{a}=\mathbf{c}_{c}(0)$. By using the bound (4.6) for $\mathbf{c}_{0}(\epsilon y)$ and the bounds (5.18) and (5.19) on the components $\mathbf{c}_{u, s}$ and $\mathbf{c}_{h}$, we derive from the integral equation (5.21) that

$$
\begin{aligned}
& \sup _{y \in\left[0, y_{0}\right]}\left\|\mathbf{c}_{c}(y)\right\|_{X_{c}} \leq K\left(\|\mathbf{a}\|_{X_{c}}+\left|\mathbf{b}\left\|_{X_{s}}+\left|\alpha_{1}\right|+\left|\alpha_{2}\right|+\epsilon M_{5} \int_{0}^{y_{0}}\right\| \mathbf{c}_{0}(y)\left\|_{X_{h}}\right\| \mathbf{c}_{c}(y) \|_{X_{c}} d y\right.\right. \\
& \left.+\epsilon y_{0} M_{6} \sup _{y \in\left[0, y_{0}\right]}\left\|\mathbf{c}_{c}(y)\right\|_{X_{c}}^{2}+\epsilon^{N+1} M_{7} \int_{0}^{y_{0}}\left\|\mathbf{c}_{0}(y)\right\|_{X_{h}} d y+\epsilon^{N+1} y_{0} M_{8} \sup _{y \in\left[0, y_{0}\right]}\left\|\mathbf{c}_{c}(y)\right\|_{X_{c}}\right)
\end{aligned}
$$

for some $M_{5}, M_{6}, M_{7}, M_{8}>0$. By the Gronwall's inequality, we have thus obtained that

$$
\begin{aligned}
\sup _{y \in\left[0, y_{0}\right]}\left\|\mathbf{c}_{c}(y)\right\|_{X_{c}} \leq & K e^{\epsilon K M_{5} \int_{0}^{y_{0}}\left\|\mathbf{c}_{0}(y)\right\|_{X_{h}} d y}\left(\|\mathbf{a}\| X_{X_{c}}+\left|\mathbf{b} \|_{X_{s}}+\right| \alpha_{1}|+| \alpha_{2} \mid\right. \\
& \left.+\epsilon^{N} M_{9}+\epsilon y_{0} M_{6} \sup _{y \in\left[0, y_{0}\right]}\left\|\mathbf{c}_{c}(y)\right\|_{X_{c}}^{2}+\epsilon^{N+1} y_{0} M_{8} \sup _{y \in\left[0, y_{0}\right]}\left\|\mathbf{c}_{c}(y)\right\|_{X_{c}}\right),
\end{aligned}
$$

for some $M_{9}>0$. Here we can use the decay bound (4.6) which implies that $\epsilon \int_{0}^{y_{0}}\left\|\mathbf{c}_{0}(y)\right\|_{X_{h}} d y \leq C$ for some $\epsilon$-independent $C>0$. By using the same bound for the exponent and letting $y_{0}=L / \epsilon^{M}$, we can see that we can choose $M \leq N+1$, where the value $M=N+1$ gives the balance of all terms in the upper bound (5.22). If arbitrary vectors $\mathbf{a}, \mathbf{b}$ and $\left(\alpha_{1}, \alpha_{2}\right)$ satisfies the bound (5.13), then we have constructed a local solution $\mathbf{c}_{c}(y)$ which satisfies the bound

$$
\sup _{y \in\left[0, L / \epsilon^{N+1}\right]}\left\|\mathbf{c}_{c}(y)\right\|_{X_{c}} \leq \tilde{C}_{c} \epsilon^{N}
$$

for some $\tilde{C}_{c}>0$. By using the bounds (5.13), (5.18), (5.19), and (5.23), we have proved the bound (5.14) for some $\epsilon$-independent constants $C_{h}, C>0$. 
Remark 7 One can prove Theorem 3 by using the contraction mapping principle and the integral formulation for the local center manifold of the system (5.5)-(5.6). This approach was undertaken in Section 4 of [5] (see their Theorem 4). We have avoided this unnecessary complication with the explicit decomposition (5.4) and analysis of the system (5.5)-(5.6) decomposed into subsystems. Similar direct methods of analysis have been applied to problems without Hamiltonian structures such as the quasilinear wave equation in [6], where an iteration scheme was employed to prove the bound on small local solutions along the local center-stable manifold.

Proof of Theorem 2: By Theorem 3, we have constructed an infinite-dimensional continuous family of local bounded solutions of the system (5.5)-(5.6) on $y \in\left[0, L / \epsilon^{N+1}\right]$ for some $\epsilon$-independent constant $L>0$. The solutions are close to the reversible homoclinic orbit of the extended coupled-mode system (4.5) in the sense of the bound (1.14). It remains to extend the local solution to the symmetric interval $y \in\left[-L / \epsilon^{N+1}, L / \epsilon^{N+1}\right]$ as the local reversible solution with the reversibility constaints (3.16). To do so, we shall consider the intersections of the local invariant manifold of the system (5.5)-(5.6) with the symmetric section $\Sigma_{r}^{\prime}$ defined by (3.17).

Since the initial data $\mathbf{c}_{c}(0)=\mathbf{a}$ in the local center-stable manifold of Theorem 3 are arbitrary, the components of a can be chosen to lie in the symmetric section $\Sigma_{r}^{\prime}$, such that

$$
\operatorname{Im}(\mathbf{a})_{m}^{+}=0, \forall m \in \mathbb{Z}_{-}^{\prime} \backslash\{n\}, \quad \operatorname{Im}(\mathbf{a})_{m}^{-}=0, \forall m \in \mathbb{Z}_{-}^{\prime} \backslash\{-n\} .
$$

This construction still leaves infinitely many arbitrary parameters for

$$
\operatorname{Re}(\mathbf{a})_{m}^{+}, \forall m \in \mathbb{Z}_{-}^{\prime} \backslash\{n\}, \quad \operatorname{Re}(\mathbf{a})_{m}^{-}, \forall m \in \mathbb{Z}_{-}^{\prime} \backslash\{-n\}
$$

to be chosen in the bound (5.13). The initial data $\mathbf{c}_{h}(0)$ and $\mathbf{c}_{s, u}(0)$ are not arbitrary since we have used the Implicit Function Theorem for the mappings (5.18) and (5.19). Therefore, we have to show that the components of $\mathbf{b}$ and $\left(\alpha_{1}, \alpha_{2}\right)$ can be chosen uniquely so that the local center-stable manifold intersects at $y=0$ with the symmetric section $\Sigma_{r}^{\prime}$.

We note that there are as many arbitrary parameters $\mathbf{s}$ and $\left(\alpha_{1}, \alpha_{2}\right)$ in the local center-stable manifold as there are remaining constraints in the set $\Sigma_{r}^{\prime}$. First, let us consider constraints in the set $\Sigma_{r}^{\prime}$ for $m \in \mathbb{Z}_{+}^{\prime}$, namely

$$
\operatorname{Re} c_{m}^{+}(0)=\operatorname{Re} c_{m}^{-}(0), \quad \operatorname{Im} c_{m}^{+}(0)=-\operatorname{Im} c_{m}^{-}(0), \quad m \in \mathbb{Z}_{+}^{\prime} .
$$

Let $\mathbf{c}_{s}=e^{y \Lambda_{s}} \mathbf{b}+\tilde{\mathbf{c}}_{s}(y)$ and rewrite the constraints in the form

$$
\operatorname{Re} b_{m}+\operatorname{Re}\left(\tilde{\mathbf{c}}_{s}\right)_{m}(0)=\operatorname{Re}\left(\mathbf{c}_{u}\right)_{m}(0), \quad \operatorname{Im} b_{m}+\operatorname{Im}\left(\tilde{\mathbf{c}}_{s}\right)_{m}(0)=-\operatorname{Im}\left(\mathbf{c}_{u}\right)_{m}(0),
$$

where all terms are of order $\mathrm{O}\left(\epsilon^{N}\right)$ and the vectors $\tilde{\mathbf{c}}_{s}(0)$ and $\mathbf{c}_{u}(0)$ depend on $\mathbf{b}$ in higher orders in $\epsilon$. By the Implicit Function Theorem, there exists a unique solution of the constraints for $\mathbf{b}$ such that $\|\mathbf{b}\|_{X_{s}}$ satisfies the bound (5.13).

Finally, let us consider constraints in the set $\Sigma_{r}^{\prime}$ for components of $\mathbf{c}_{h}$, namely

$$
\operatorname{Im} c_{n}^{+}(0)=0, \quad \operatorname{Im} c_{-n}^{-}(0)=0 .
$$

Let $\mathbf{c}_{h}=\alpha_{1} \mathbf{s}_{1}+\alpha_{2} \mathbf{s}_{2}+\tilde{\mathbf{c}}_{s}(y)$ and note that $\mathbf{s}_{1}$ and $\mathbf{s}_{2}$ violate the constraints (5.28). Let $\mathbf{a}$ and $\mathbf{b}$ be chosen so that $\mathbf{c}(0)$ belongs to the set $\Sigma_{r}^{\prime}$. By a construction of the vector field in the system (5.16), if $\mathbf{c}(y)$ lies in the domain $D_{r}^{\prime}$ of reversible solution and $\alpha_{1}=\alpha_{2}=0$, then $\mathbf{G}_{Q, R}^{*}=\mathbf{G}_{Q, R}$ on $y \in\left[-y_{0}, y_{0}\right]$ 
and the global solution $\tilde{\mathbf{c}}_{s}(y)$ constructed in Theorem 3 intersects the set $\Sigma_{r}^{\prime}$ at $y=0$. Therefore, the choice $\alpha_{1}=\alpha_{2}=0$ satisfies the constraints (5.28) identically.

We have thus constructed a family of reversible solutions in the symmetric interval $y \in\left[-L / \epsilon^{N+1}, L / \epsilon^{N+1}\right]$ while preserving the bound (1.14). Tracing the coordinate transformations used in our analysis back to the original variable $\psi(x, y)$, we have thus completed the proof of Theorem 2 .

\section{Discussion}

We have proved that a moving gap soliton of the Gross-Pitaevskii equation (1.1) with the periodic potential $V(x)$ is surrounded by the oscillatory tails which are bounded on finite intervals of the spatial scale. Because the center manifold is infinite-dimensional with the sign-indefinite Hamiltonian function, we are not able to exclude the polynomial growth of the oscillatory tails in the far-field regions. This construction of traveling solutions on a finite spatial scale is related with the finite-time applicability of the coupled-mode equations (1.5) for the Cauchy problem associated with the Gross-Pitaevskii equation (1.1) [12].

It would have been a drastic improvement to the constructed theory if we could extend the analysis of oscillatory tails to the infinite spatial scale by proving existence of global solutions with a single bump and bounded oscillatory tails. In many problems with finite-dimensional center manifolds associated with semi-simple purely imaginary eigenvalues, such constructions of global center-stable manifolds are possible and the proof of persistence of bounded solutions with oscillatory tails can be developed [3].

We will show that the basic evolution models for moving gap solitons in periodic potentials exhibit infinite-dimensional center manifolds in the spatial dynamics formulation. In particular, we can think of three possible generalizations of the Gross-Pitaevskii equation (1.1), given by the complex-valued Klein-Gordon equation

$$
E_{t t}-E_{x x}=V(x) E+\sigma|E|^{2} E,
$$

the regularized Gross-Pitaevskii equation

$$
i E_{t}=-E_{x x}+i E_{x x t}+V(x) E+\sigma|E|^{2} E,
$$

and the discrete Gross-Pitaevskii equation

$$
i \dot{E}_{n}=-E_{n+1}-E_{n-1}+V_{n} E_{n}+\sigma\left|E_{n}\right|^{2} E_{n} .
$$

When $V(x) \equiv 0$ or $V_{n} \equiv 0$, the spectrum of the linear part of the Klein-Gordon equation (6.1) is unbounded from both above and below, while that of the regularized and discrete Gross-Pitaevskii equations (6.2) and (6.3) is bounded from both above and below. We look at the traveling solutions of these equations in the form

$$
E(x, t)=\sum_{m \in \mathbb{Z}^{\prime}} \psi_{m}(y) e^{\frac{i m x}{2}-i \omega t}, \quad y=x-c t,
$$

for the linear limit $\sigma=0$ with no potential $V(x) \equiv 0$. (In the case of the lattice equation (6.3), we use the traveling ansatz (6.4) at $x=n$ for $n \in \mathbb{N}$.) As a result, we obtain uncoupled linear ODEs or 
differential advance-delay equations for amplitudes $\left\{\psi_{m}(y)\right\}_{m \in \mathbb{Z}^{\prime}}$ which are solved with the substitution $\psi_{m}(y)=e^{\kappa y}, \forall m \in \mathbb{Z}^{\prime}$. All roots $\kappa$ are found from the following characteristic equations

$$
\begin{aligned}
\left(1-c^{2}\right) \kappa^{2}+i(m-2 c \omega) \kappa+\omega-\frac{m^{2}}{4} & =0, \\
-i c \kappa^{3}+(\omega-1+m c) \kappa^{2}+i\left(c-m+m \omega+\frac{m^{2} c}{4}\right) \kappa-\omega+\frac{m^{2}}{4}(1-\omega) & =0, \\
\omega-i c \kappa+2 \cosh \left(\kappa+\frac{m}{2}\right) & =0,
\end{aligned}
$$

which correspond to the three relevant models (6.1)-(6.3). It is easy to see that the characteristic equations (6.6) and (6.7) have at least one purely imaginary root $p$ for any $m \in \mathbb{Z}^{\prime}$, while the characteristic equation (6.5) has two purely imaginary roots $p$ for sufficiently large values of $|m|$ on $m \in \mathbb{Z}^{\prime}$. Therefore, the dimension of the center manifold associated with the linearized system at the zero solution for $V(x) \equiv 0$ is infinite in all three models (6.1) -6.3 .

Acknowledgement. The work of D. Pelinovsky is supported by the Humboldt Research Foundation. The work of G. Schneider is partially supported by the Graduiertenkolleg 1294 "Analysis, simulation and design of nano-technological processes" granted by the Deutsche Forschungsgemeinschaft (DFG) and the Land Baden-Württemberg.

\section{References}

[1] G. Alfimov and V.V. Konotop, "On the existence of gap solitons", Physica D 146, 307-327 (2000)

[2] M. Chugunova and D. Pelinovsky, "Block-diagonalization of the symmetric first-order coupledmode system", SIAM J. Appl. Dyn. Syst. 5, 66-83 (2006)

[3] G. Iooss and E. Lombardi, "Polynomial normal forms with exponentially small remainder for analytic vector fields", J. Diff. Eqs. 212, 1-61 (2005)

[4] R.H. Goodman, M.I. Weinstein, and P.J. Holmes, "Nonlinear propagation of light in onedimensional periodic structures", J. Nonlinear. Science 11, 123-168 (2001)

[5] M.D. Groves and G. Schneider, "Modulating pulse solutions for a class of nonlinear wave equations", Commun. Math. Phys. 219, 489-522 (2001)

[6] M.D. Groves and G. Schneider, "Modulating pulse solutions for quasilinear wave equations", J. Diff. Eqs. 219, 221-258 (2005)

[7] "Emergent Nonlinear Phenomena in Bose-Einstein Condensates", Eds. P.G. Kevrekidis, D.J. Franzeskakis, and R. Carretero-Gonzalez (Springer-Verlag, New York, 2007)

[8] Yu.S. Kivshar and B.A. Malomed, "Dynamics of solitons in nearly integrable systems", Rev. Mod. Phys. 61, 763 - 915 (1989)

[9] A. Pankov, "Periodic nonlinear Schrdinger equation with application to photonic crystals", Milan J. Math. 73, 259-287 (2005) 
[10] D. Pelinovsky and G. Schneider, "Justification of the coupled-mode approximation for a nonlinear elliptic problem with a periodic potential", preprint (2007).

[11] A. Sánchez and A.R. Bishop, "Collective coordinates and length-scale competition in spatially inhomogeneous soliton-bearing equations", SIAM Review 40, 579-615 (1998).

[12] G. Schneider and H. Uecker, "Nonlinear coupled mode dynamics in hyperbolic and parabolic periodically structured spatially extended systems", Asymp. Anal. 28, 163-180 (2001)

[13] C.M. de Sterke and J.E. Sipe, "Gap solitons", Progress in Optics, 33, 203 (1994) 